\title{
Gastroprotective and anti-Helicobacter pylori potential of herbal formula HZJW: safety and efficacy assessment
}

\author{
Jian-Hui Xie ${ }^{1 \dagger}$, Yun-Long Chen ${ }^{1 \dagger}$, Qing-He Wu' ${ }^{1}$, Jun Wu ${ }^{3}$, Ji-Yan Su${ }^{1}$, Hong-Yin Cao ${ }^{1}$, Yu-Cui Li ${ }^{1}$, Yi-Sheng Li ${ }^{4}$,
} Jin-Bin Liao ${ }^{1}$, Xiao-Ping Lai ${ }^{1,2}$, Ping Huang ${ }^{1 *}$ and Zi-Ren Su ${ }^{1,2^{*}}$

\begin{abstract}
Background: A traditional Chinese Medicine (TCM) formula, HZJW, has been applied in clinics in China for gastrointestinal disorders. However, the therapeutic mechanism underlying its efficacy and safety remained to be defined. The present investigation was undertaken to evaluate the formula HZJW for its gastroprotective potential, possible effect on Helicobacter pylori along with safety to justify its anti-ulcer action and safe clinical application.

Methods: The gastroduodenal cytoprotective potential was evaluated in rodent experimental models ( $\mathrm{HCl} /$ Ethanol and NSAID-induced ulcer protocols). The anti-H. pylori property was assessed by agar dilution assay in vitro and analysis in vivo including rapid urease test, immunogold test and histopathology. For toxicity assessment, acute toxicity study was performed according to fixed dose procedure with a single oral administration of HZJW to mice. In the oral chronic toxicity, rats (80 males, 80 females) were administrated HZJW orally in 0, 1000, 2500, or $5000 \mathrm{mg} / \mathrm{kg} /$ day doses for 26 weeks ( $n=40 /$ group of each sex). Clinical signs, mortality, body weights, feed consumption, ophthalmology, hematology, serum biochemistry, gross findings, organ weights and histopathology were examined at the end of the 13- and 26-week dosing period, as well as after the 4-week recovery period.

Results: In the HCl/Ethanol-induced ulcer model, it was observed that oral administration with HZJW (260, 520 and $1040 \mathrm{mg} / \mathrm{kg}$ ) and ranitidine $(250 \mathrm{mg} / \mathrm{kg})$ significantly reduced the ulcerative lesion index (116.70 $\pm 36.4,102.20 \pm 18.20$, $84.10 \pm 12.1$ and $73.70 \pm 16.70)$ in a dose-dependent manner, respectively, with respect to control group (134.10 \pm 31.69$)$. Significant inhibition was also observed in ulcerative index from aspirin-induced ulcer model, with decreases of $35.40 \pm$ $5.93,31.30 \pm 8.08,26.80 \pm 8.27$ and $20.40 \pm 6.93$ for the groups treated with HZJW and ranitidine, in parallel to controls (41.60 \pm 10.80 ). On the other hand, treatment with HZJW efficaciously eradicated H. pylori in infected mice in rapid urease test (RUT) and immunogold antibody assay, as further confirmed by reduction of $\mathrm{H}$. pylori presence in histopathological analysis. In the in vitro assay, MICs for HZJW and amoxicillin (positive control) were $125 \mathrm{and} 0.12 \mathrm{\mu g} / \mathrm{mL}$ respectively. The $\mathrm{LD}_{50}$ of HZJW was over $18.0 \mathrm{~g} / \mathrm{kg}$ for mice. No drug-induced abnormalities were found as clinical signs, body weight, food consumption, hematology, blood biochemistry, ophthalmology and histopathology results across three doses. No target organ was identified. The No Observed Adverse Effect Level (NOAEL) of HZJW was determined to be $5,000 \mathrm{mg} / \mathrm{kg} /$ day for both sexes, a dose that was equivalent to 50 times of human dose.

(Continued on next page)
\end{abstract}

\footnotetext{
* Correspondence: hping331@126.com; suziren@gzucm.edu.cn

${ }^{\dagger}$ Equal contributors

${ }^{1}$ School of Chinese Materia Medica, Guangzhou University of Chinese Medicine, Guangzhou 510006, P. R. China

${ }^{2}$ Dongguan Mathematical Engineering Academy of Chinese Medicine, Guangzhou University of Chinese Medicine, Dongguan 523808, P. R. China Full list of author information is available at the end of the article
} 
(Continued from previous page)

Conclusions: These results suggested the efficacy and safety of HZJW in healing peptic ulcer and combating H. pylori, which corroborated their conventional indications and contributed to their antiulcer pharmacological validation, lending more credence to its clinical application for the traditional treatment of stomach complaints symptomatic of peptic ulcer disease (PUD). HZJW might have the potential for further development as a safe and effective alternative/complementary to conventional medication in treating gastrointestinal (Gl) disorders.

Keywords: HZJW, TCM, Cytoprotective, Helicobacter pylori, Gastroduodenal ulcer, Safety

\section{Background}

Gastric and duodenal ulcers (peptic ulcers) are the most common gastrointestinal disorder that occur in clinical practice and are currently considered as a progressive global health problem. The ulcers that affect the gastrointestinal system are normally provoked by an imbalance between aggressive and protective factors in the stomach [1]. Furthermore, it is also widely accepted that most peptic ulcers are associated with Helicobacter pylori infection and eradiation of this organism leads to enhanced ulcer healing and much less chance of ulcer recurrence [2,3].The current medicinal treatment of peptic ulcer is generally based on triple therapy regimen, inhibition of gastric acid secretion by histamine $\mathrm{H}_{2}$-antagonists, proton pump inhibitors, as well as on mucosal protective therapy provided by sucralfate and bismuth [4]. However, the treatment is complicated and of high cost, requiring a minimum of two antibiotics in combination with gastric acid inhibitors, which often causes nausea, antibiotic resistance, recurrence and other adverse effects [5-7]. In view of their various harmful adverse effects and the prevalence of antibiotic-resistant $H$. pylori strains, the search for safe and effective non-antibiotic agents is essential. In recent years, active researches have rekindled interest in natural drugs possessing these activities, and there has been an increased inclination towards herbal formulations, which are widely appreciated by the population especially in oriental countries.

For centuries, herbals have been used traditionally for the treatment of a wide range of ailments, including gastrointestinal (GI) disorders [8,9], such as dyspepsia, gastritis and peptic ulcer disease (PUD). In China, a variety of Chinese medicinal herbs have been prescribed to attenuate or eradicate gastritis-like disorders and achieved good effects. HZJW, composed of 12 medicinal herbs, is a Chinese herbal formula based on a famous proved recipe proposed by Prof. Honglin Xing (Table 1). HZJW had clinical efficacy of reinforcing spleen and stomach to clear away heat and eliminate dampness, promoting $q i$ and blood circulation to relieve pain and flatulence, and had been demonstrated to possess pronounced effectiveness and safety for the treatment of gastrointestinal disease in clinical practice $[10,11]$. Traditionally, these twelve herbs with well-established histories of application are commonly prescribed together with other herbs in herbal formulas to treat gastrointestinal disorders by Chinese medicine practitioners. According to the theory of TCM, a reasonable combination of medicinal herbs was used to enhance the desired action and eliminate possible side effects. Corydalis Rhizoma, Coptidis Rhizoma and Alpinia officinarum, important components of HZJW, were proved to be effective in the prevention of $H$. pylori infection [12,13]. Berberine and its protoberberine alkaloids palmatine, coptisine and aporphinoid alkaloid of magnoflorine have been confirmed to be the major pharmacologically active constituents of Coptidis Rhizoma against H. pylori [14-16]. Berberin, one of the chemical marker of this formula, was also found to possess simultaneous gastrointestine protective and anti-inflammatory activities [15].

In clinical practice in China, we have successfully applied this recipe for treating patients with gastrointestinal disorders. Nevertheless, the evidence-based mechanism of the reputed efficacy remains elusive. No standard preclinical toxicity data in any animal species are currently available to assess its potential toxic effects. The present research was to explore possible mechanisms underlying

Table 1 Composition of HZJW

\begin{tabular}{|c|c|c|}
\hline Linnean classification & Botanical origin & Weight ratio \\
\hline Macrocephalae Rhizoma & $\begin{array}{c}\text { Atractylodes macrocephala } \\
\text { Koidz. }\end{array}$ & 0.2 \\
\hline Scutellariae Barabtae Herba & Scutellaria barbata D. Don & 0.15 \\
\hline Corydalis Rhizoma & $\begin{array}{c}\text { Corydalis yanhusuo } \\
\text { W.T. Wang }\end{array}$ & 0.12 \\
\hline Curcumae Radix & $\begin{array}{l}\text { Curcuma wenyujin Y.H. } \\
\text { Chen et C.Ling }\end{array}$ & 0.12 \\
\hline $\begin{array}{l}\text { Cynanchi Paniculati } \\
\text { Radix et Rhizoma }\end{array}$ & $\begin{array}{c}\text { Cynanchum paniculatunm } \\
\text { (Bge.) Kitag. }\end{array}$ & 0.12 \\
\hline Alpiniae Officmarum Rhizoma & Alpinia officinarum Hance & 0.03 \\
\hline Coptidis Rhizoma & Coptis chinensis Franch. & 0.06 \\
\hline Perillae Fructus & Perilla frutescens (L.) Britt. & 0.12 \\
\hline Taraxact Herba & $\begin{array}{c}\text { Taraxacum mongolicum } \\
\text { Hand. Maza. }\end{array}$ & 0.15 \\
\hline Typhae Pollen & Typha angustifolia L. & 0.12 \\
\hline Hedyotis diffusa Willd & $\begin{array}{l}\text { Olydenlandia diffusa } \\
\text { (Wild.) Roxb. }\end{array}$ & 0.3 \\
\hline Herba Eupatorii & Eupatorium fortunei Turcz. & 0.15 \\
\hline
\end{tabular}


the pharmacological action of HZJW and unravel its comprehensive toxicological properties. In view of the critical role of cytoprotective and anti- $H$. pylori activity in the management of peptic ulcer, the development of a single preparation endowed with both properties will be a welcome contribution. Firstly, we assessed the gastroprotective activity via different rodent experimental models ( $\mathrm{HCl} /$ Ethanol and NSAID-induced ulcer protocols). Secondly, we determined the anti-H. pylori effect of HZJW in vitro by agar dilution assay and in vivo via rapid urease test, immunogold assay and histopathological examination. Thirdly, we undertook an acute toxicity appraisal in Kun-ming mice and a 26-week chronic toxicological study on Sprague-Dawley rats to characterize its possible toxicity. The present scheme should contribute to an improved understanding of possible mechanisms of HZJW and, also valuable information of toxicity to direct its clinical application.

\section{Methods}

\section{Sources and authentication of herbs}

All the herbs of HZJW formula were obtained from Yifang Chinese Material Medica Business Department, Yulin City, all of which are authenticated by Prof. Lai Xiaoping at Guangzhou University of Chinese Medicine. The authenticated voucher specimens (Voucher \#20090924) were kept in School of Chinese Materia Medica, Guangzhou University of Chinese Medicine (GUCM). Assurance of quality control for all the materials was validated according to Pharmacopoeia of the People's Republic of China [17].

\section{Preparation of HZJW}

HZJW was composed of 12 medicinal herbs as shown in Table 1. Coptidis Rhizoma and Corydalis Rhizoma were ground to obtain fine powder while others ground to be coarse powder. Cynanchi Paniculati Radix et Rhizoma was distilled with water $(1: 8, w / v)$ for $4 \mathrm{~h}$ and $30 \% \mathrm{NaCl}$ was added into the obtained distillate to yield salt-outings. After filtration, the residues of Cynanchi Paniculati Radix et Rhizoma and the other nine medicinal herbs (except Coptidis Rhizoma and Corydalis Rhizoma) were extracted with water $(1: 10, \mathrm{w} / \mathrm{v})$ twice, $1 \mathrm{~h}$ each, of which Scutellariae Barabtae Herba was added post boiling. The resulting supernatant was concentrated to a relative density (RD) of $1.20\left(60^{\circ} \mathrm{C}\right)$, mixed together with the abovementioned fine powder thoroughly, and then dried out under vacuum. Finally, the above dry extract was blended thoroughly with the salt-outings and an appropriate amount of microcrystalline cellulose to produce HZJW. The yield of HZJW extract was $31.75 \%(\mathrm{w} / \mathrm{w})$ compared with the original herbs. Chemical profile of HZJW was analyzed by HPLC (see Additional file 1: Figure S1). A voucher specimen was deposited at GUCM, with the registration number
20091102. HZJW was stored at $4^{\circ} \mathrm{C}$ and diluted to the desired concentrations in distilled water at the time of administration.

\section{Bacterial strains and cultivation}

Helicobacter pylori strain ATCC 43504 (Vac A and Cag $A$ positives) employed for the assay was obtained from American Type Culture Collection (Rockville, MD), and stored at $-80^{\circ} \mathrm{C}$ in Muller-Hinton broth (OXOID) containing $15 \%(\mathrm{w} / \mathrm{v})$ glycerol until experimentation. Frozen H. pylori isolate was thawed and grown on Brucella agar supplemented with bovine serum albumin (BSA) for $72 \mathrm{~h}$ at $37^{\circ} \mathrm{C}$ under a microaerophilic atmosphere $\left(85 \% \mathrm{~N}_{2}\right.$, $\left.10 \% \mathrm{CO}_{2}, 5 \% \mathrm{O}_{2}\right)$ and $98 \%$ humidity. Each plate was swabbed with a sterile cotton-tipped applicator, and the tested organism inocula used for the dilution tests were prepared by suspending $72 \mathrm{~h}$ colonies in $2 \mathrm{~mL}$ of sterile distilled water (DW) to obtain turbidity equivalent to a 2.0 McFarland standard ( $\left.10^{8} \mathrm{CFU} / \mathrm{mL}\right)$.

\section{Experimental animals and maintenance}

Sprague-Dawley (SD) rats $(180 \pm 20 \mathrm{~g})$, Balb/c mice $(18 \pm 2 \mathrm{~g})$, Kun-ming $(\mathrm{KM})$ mice $(18 \pm 2 \mathrm{~g})$ of both sexes at the initiation of treatment were obtained from the Medical Experimental Animal Center of Guangzhou University of Chinese Medicine and Evaluation and Research Center for Toxicology Institute of Disease Control and Prevention PLA. Licences for rats and mice were SCXK (YUE) 2008-0020, 2009-0210 and SCXK (JUN)2007-004, respectively. For the experiment, the animals were transferred for the laboratory and submitted to adaptation by period of 7 days. They were acclimatized under controlled temperature $\left(25 \pm 2^{\circ} \mathrm{C}\right)$ and humidity (50-70\%) on a 12-h light/12-h dark cycle (artificial lighting from 08:00 to 20:00) and had free access to standard chow and drinking water. In all experiments, the animals were kept in cages with raised, wide-mesh floors to prevent coprophagy. The experimental protocols involved were in accordance to the rules and guidelines of the Experimental Animal Center of Guangzhou University of Chinese Medicine and, approved by the Animal Care and Use Ethics Committee of our institution, with reference to European Community guidelines for the use of experimental animals.

\section{Chemicals and drugs}

Aspirin was obtained from Bayer HealthCare AG (Lot. BTA8RR3), dexamethasone was obtained from Zhejiang Xianju pharmaceutical Co., LTD (Lot. 091032);ranitidine was purchased from $\mathrm{O}$ tevez (foshan) pharmaceutical Co., LTD (Lot. 0908521); amoxicillin was from Zhuhai federal pharmaceutical Co., LTD (Lot. 00800208). Azithromycin was purchased from Suzhou Changzheng Hinkay pharmaceutical Co., LTD (Lot. 100604); gentamycin 
was obtained from Henan topfond pharmaceutical Co., LTD (Lot. 100630950); ampicillin was obtained from Shandong Lukang pharmaceutical Co., LTD (Lot. L100708). Formaldehyde and phenol red were from Guangzhou Chemical Reagent Factory (Lot. 20100302 \& 20091021). Hydrochloric acid, sodium hydroxide and alcohol were purchased from Guangzhou chemical reagent factory (Lot. 20091014, 20090401 \& 20090304). H. pylori urease Immunogold Testing kit was from Beijing Tian Hong Sig biotechnology Co., Ltd (Lot. 010652); H. pylori infection test paper (Rapid Urease Test, RUT) was from Zhuhai Kedi science and technology development Co., Ltd (Lot. 110401). Scutellarin (Lot. 110842-200605), berberine hydrochloride (Lot. 110713-200910) and paeonol (Lot. 110708-200505) were purchased from National Institute of Food and Drug Control (Beijing, China) with purity over 98\% based on HPLC analysis. HPLC-grade ethanol and acetonitril were obtained from Honeywell (Honeywell, USA). All reagents were at least of analytic grade and applied according to the specific instruction manual.

\section{$\mathrm{HCl} /$ Ethanol-induced ulcerogenesis}

The experiment was carried out according to the method of Morimoto et al. [18], with the following modifications. Rats of each sex were randomly divided into five groups of ten animals each. The first group was given $1 \mathrm{~mL}$ of vehicle (normal saline), and the second group was treated with ranitidine $(250 \mathrm{mg} / \mathrm{kg}$, p.o.). The remaining groups received 260, 520 and $1040 \mathrm{mg} / \mathrm{kg}$ (p.o.) of HZJW respectively. All the treatments were administered daily for 3 consecutive days. All rats were subjected to abrosia $24 \mathrm{~h}$ prior to the ulcerogenic challenge. On the $4^{\text {th }}$ day, one hour after the last administration, all rats received an oral dose of $1 \mathrm{~mL}$ of $0.15 \mathrm{M}$ $\mathrm{HCl}$ in $60 \%$ ethanol to induce gastric ulcer. One hour post ulcerogenic challenge, all animals were sacrificed by cervical dislocation, and stomach of each rat was removed and inflated with $10 \mathrm{~mL}$ of $1 \%$ buffered formalin solution to fix for $10 \mathrm{~min}$. Subsequently, each stomach was incised along the greater curvature and rinsed with normal saline to remove stomach contents, then the lengths of the necrotizing lesions were measured under a dissecting microscope, to access the formation of ulcers (hemorrhagic lesions).The maximum length of each lesion was determined and the sum of lengths of all lesions $(\mathrm{mm})$ for each stomach was expressed as the ulcer index (UI), and the inhibition percentage was calculated by the following formula:

$$
\text { Inhibition }(\%)=\left[\left(\mathrm{UI}_{\text {control }}-\mathrm{UI}_{\text {treat }}\right) / \mathrm{UI}_{\text {control }}\right] \times 100 \%
$$

\section{Nonsteroidal anti-inflammatory drug (NSAID)-induced ulcer}

The experiment was carried out according to the method by Nwafor et al. [19] with a few modifications. Rats of either sex were randomly divided into five groups of ten animals each. The first group was given $1 \mathrm{~mL}$ of vehicle (normal saline), and the second group was treated with ranitidine $(250 \mathrm{mg} / \mathrm{kg}$, p.o.). The remaining groups received 260, 520 and $1040 \mathrm{mg} / \mathrm{kg}$ (p.o.) of HZJW respectively. All the treatments were administered daily for 3 consecutive days. All rats were subjected to fast $24 \mathrm{~h}$ before the ulcerogenic challenge. On the $4^{\text {th }}$ day, one hour after the last administration, all the rats received an oral dose of aspirin $(100 \mathrm{mg} / \mathrm{kg})$ to induce gastric ulcer. Seven hours after the aspirin challenge, all animals were sacrificed by cervical dislocation, and stomach of each rat was excised and inflated with $10 \mathrm{~mL}$ of $1 \%$ buffered formalin solution to fix for $10 \mathrm{~min}$. Thereafter, the greater curvature of each stomach was incised, and the extent of gastric damage in the glandular region was evaluated according to the ulcerative lesion index and inhibition percentage as described above.

\section{In vitro anti-H. pylori assay}

Agar dilution test was employed to analyze the susceptibility of reference strain $H$. pylori to HZJW in two-fold serial dilution in the range of $500-0.5 \mathrm{mg} / \mathrm{mL}$. H. pylori were cultivated for 72 hours on blood agar, harvested and suspended in Brucella broth at a final concentration of $2.48 \times 10^{9} \mathrm{CFU} / \mathrm{mL}$. Thereafter, a volume of $100 \mu \mathrm{L}$ bacterial suspension was inoculated in the Brucella medium containing HZJW of serial decreasing concentrations (500-0.5 mg/mL). In addition to the tested agent, amoxicillin was employed as the positive control. Under the same condition, surfaces that were inoculated with a suspension of $H$. pylori without any tested materials and non-inoculated media were used as controls. Inocula were incubated at $37^{\circ} \mathrm{C}$ under a microaerophilic atmosphere for 72 hours. Minimum inhibitory concentration (MIC), the lowest concentration that inhibits the visible growth of bacterium was determined. This experiment was performed in triplicate.

\section{In vivo anti-H. pylori assay}

$\mathrm{Balb} / \mathrm{c}$ mice of either sex (except the normal group that was given normal saline) were pretreated with an oral dose of $0.5 \mathrm{~mL}$ antibiotic mixture (ampicillin $10 \mathrm{mg} / \mathrm{mL}$, gentamicin $1.2 \mathrm{mg} / \mathrm{mL}$, azithromycin $10 \mathrm{mg} / \mathrm{mL}$ ) for 3 consective days, to make sure that they were free from any $H$. pylori-like organisms that could have been acquired through natural infection. On the 4th day, mice were intragastricly administrated with $0.5 \mathrm{~mL}$ freshly prepared suspension of $H$. pylori $\left(10^{8} \mathrm{CFU} / \mathrm{mL}\right)$ daily for 7 days. During this interval, mice were deprived of chew supplement but free access to drinking water was 
maintained for $12 \mathrm{~h}$ each day. On the second day following the last inoculation, the infected animals were randomly grouped according to the following doses: 520, 1040 and $2080 \mathrm{mg} / \mathrm{kg} \mathrm{HZJW}$, and were treated for 8 successive weeks (one administration per day), while the control group was given $20 \mathrm{mg} / \mathrm{kg}$ normal saline. Amoxicillin, suspended in $0.5 \% \mathrm{w} / \mathrm{v} \mathrm{CMC}$, was used as reference drug, and was administered orally to the mice for 8 successive weeks at a dose of $670 \mathrm{mg} / \mathrm{kg}$ body weight, which was approximately 10 times of the maximum recommended human dose. After the last administration, all mice were fasted for $24 \mathrm{~h}$, blood samples were collected from retro-orbital and sacrificed by cervical dislocation. Serum was prepared and subjected to $H$. pylori urease Immunogold Testing kit to assess $H$. pylori clearance. All procedures were performed as described according to the manufacturers' recommendation in the kit manuals.

At the same time, the stomach was excised, and the gastric mucosa tissue was submitted to rapid urease test (RUT). In brief, with the aid of tweezers, a fragment of gastric tissue was inserted in the centre of a minitube containing urease gel. Inoculation times were recorded. The minitubes were kept at room temperature and the colour change was evaluated within $3 \mathrm{~min}$. Test result was considered strong positive if an alkaline reaction had developed (from yellow to dark pink within the tissue margin) in $1 \mathrm{~min}$, and weak positive if in $3 \mathrm{~min}$, while negative if the colour failed to exhibit any colour variation within $3 \mathrm{~min}$ in the medium (yellow or light orange). The rest of the stomach tissues were fixed with a $10 \%$ formalin solution, dehydrated, embedded in paraffin, sectioned, deparaffinized, and stained with carbolic acid and basic fuchsin for $H$. pylori detection.

\section{Acute toxicity test of HZJW}

Acute oral toxicity study was performed according to fixed dose procedure. Two experimental groups of mice (10 mice of each sex in each group) were treated orally with a single dose of 0 and $18.0 \mathrm{~g} / \mathrm{kg}$ body weight. Animals receiving the vehicle (saline) served as control. Animals were observed individually at least once during the first $30 \mathrm{~min}$ after administration, periodically during the first $24 \mathrm{~h}$ (with special attention during the first $4 \mathrm{~h}$ ) and daily thereafter for a period of 14 days. The observation principally included changes in skin and fur, eyes and mucous membrane (nasal), autonomic changes (salivation, lacrimation, perspiration, piloerection, urinary volume, and defecation) and alterations of the central nervous system (ptosis, drowsiness, gait, tremors and convulsion). Food and water were provided throughout the experiment. For 14 days the animals were weighed and the number of deaths noted.

\section{Experimental schedule for general toxicity of HZJW}

To generally detect potential long-term, repeat-dose toxicity of HZJW and thereby define the characteristic, extent, dose and time-dependent relationship, as well as target organs and tissues of potentially toxic effects, 80 male and 80 female SD rats were assigned randomly and evenly to 4 experimental groups. The animals were separated by gender and housed five in each cage. Group I animals (control) were orally administered with distilled water throughout the course of the study. Animals in Groups II (1000 mg/kg body weight/day), III (2500 mg/ kg body weight/day), IV (5000 mg/kg body weight/day) received orally administered HZJW dissolved in distilled water once-daily for a period of 13 or 26 weeks. The dosages selected in the present study were based on existing data on the effective dose, results of acute toxicity study of HZJW in mice, and suggested human dose (equivalent to 10, 25, and 50 times of the normal human dose in clinical prescription, respectively). Clinical signs (general behavior, fur condition, breathing and nose conditions, eyes and oral secretions, urine and faeces), toxic reactions, and mortality were monitored daily after the initiation of HZJW treatment. Body weight, food and water consumption were recorded once a week. After 13 weeks, 5 rats per each sex in each group were sacrificed. Another 10 rats per each sex in each group were sacrificed at the end of the 26 th week. The remaining rats were sacrificed after 4 weeks of recovery. The animals of the recovery groups were observed for reversibility, persistence and delayed occurrence of toxic effects. All examinations were conducted as described above.

At the end of the tested period, all animals were sacrificed and subjected to hematology and clinical chemistry assays, ophthalmic testing (cornea, conjunctiva, iris, pupil, atria, len, fundus, eyelid), necropsy examination, organ weighing, and histopathologic examination. This toxicity study was carried out in compliance with the Testing Guidelines for Safety Evaluation of Drugs (Notification [Z] GPT3-1 issued by China Food and Drug Administration (SFDA) on March 2005) and Good Laboratory Practice Regulations for Nonclinical Laboratory Studies.

\section{Clinical observation and feed consumption}

The animals were observed daily prior to and following administration for signs of toxicity and mortality throughout the experimental period. Detailed clinical signs were assessed and recorded, including changes in skin and fur, eyes and mucous membranes, manure, psyche states and behavior patterns, etc. The body weight was measured at the initiation of treatment and once a week during the treatment period thereafter. The amounts of feed were weighed before they were supplied to each cage and their remnants were measured the next day. The differences 
were calculated and regarded as daily feed consumption (g/animal/day).

\section{Haematological and biochemical parameters}

Samples of blood were obtained from all animals on Week 13 and Week 26 of the investigation, and after recovery. All rats were fasting but allowed access to water ad libitum for more than $12 \mathrm{~h}$ prior to blood sample collecting. Blood samples were collected into two tubes: (1) heparinized centrifuge tubes and (2) dry nonheparinized centrifuge tubes. The heparinized blood was used for a hematological determination, which include red blood cell (RBC), hemoglobin (HGB), hematocrit (HCT), mean corpuscular volume (MCV), mean corpuscular HGB $(\mathrm{MCH})$, mean corpuscular HGB concentration $(\mathrm{MCHC})$, red cell distribution width (RDW), platelets (PLT), mean platelet volume (MPV), platelet distribution width (PDW), white blood cell (WBC) counts, reticulocytes (RET), neutrophils (NE), lymphocytes (LYM), monocytes (MON), eosinophils (EOS), and basophils (BAS) with a MEK-7222K Hematology analyzer (Nihon Kohden, Japan) according to the manufacturer's operator manual. Plasma was isolated and used to determine the prothrombin time (PT) using a coagulometer (CA-50; Sysmex, Japan).

The non-heparinized blood was allowed to coagulate before being centrifuged and the serum separated. Serum was analyzed for changes in biochemistry using an automatic biochemical analyzer (RA-1000, Technico, USA), which measured aspartate aminotransferase activity (AST), alanine aminotransferase activity (ALT), alkaline phosphatase activity (ALP), albumin (ALB), total protein (TP), total cholesterol (CHOL), creatine phosphokinase activity (CPK), total bilirubin (T-BIL), direct bilirubin (D-BIL), creatinine (Cre), triglycerides (TG), urea (Ure) and glucose (GLU). The concentrations of sodium ions $\left(\mathrm{Na}^{+}\right)$, potassium ions $\left(\mathrm{K}^{+}\right)$and chloride ions $\left(\mathrm{Cl}^{-}\right)$ were measured with an electrolyte autoanalyzer (EasyLyte $\mathrm{Na}^{+} / \mathrm{K}^{+} / \mathrm{Cl}^{-}$Analyzer, MEDICA, USA). All parameters of blood chemistry and hematology were measured following standard procedures.

\section{Organ weights, gross necropsy and histopathological examination}

All animals were fasted overnight before scheduled necropsies. A complete necropsy was conducted on all animals and consisted of an external examination, including the identification of all clinically recorded lesions, and a detailed internal examination. The rats were anesthetized with diethyl ether and sacrificed by decapitation after blood collection from the abdominal aorta. After dissection to remove fat and connective tissue, the following organs were carefully dissected out and weighed: brain (cerebrum, cerecellum and brainstem), thymus glands, heart, lungs, liver, spleen, kidneys, adrenal glands, testis/ovary and epididymis/uterus. The relative organ weight was calculated based on the terminal body weight before fixation.

The following organs of all animals were fixed in 10\% neutral formalin: brain, thoracic spinal cord, thyroid glands, Gley's glands, pituitary gland, thymus, adrenal glands, esophagus, salivary glands, stomach, small intestine (duodenum, jejunum and ileum), large intestine (cecum, colon and rectum), pancreas, spleen, lungs, trachea, kidneys, liver, heart, aorta, bladder, testes, epididymides, ovaries, uterus, prostate gland, vagina, mammary gland, sciatic nerve, urinary mesenteric lymph node, sternum, sub-mandibular lymph nodes, bone marrow.

The fixed organs of the vehicle control and high dose group and any organs from the other groups that displayed gross abnormalities were subjected to histopathological examination. These samples were Paraffin-embedded, microsectioned at a nominal thickness of $4 \mu \mathrm{m}$ and then stained with hematoxylin and eosin for histopathological examination.

\section{Statistical analysis}

The data obtained by the various parameters was statistically evaluated by one way analysis of variance (ANOVA), and presented as means \pm standard deviation (S.D.) for the indicated number of independently performed experiments. The body and organ weight, food and water consumption, hematological parameters, and blood biochemical parameters were analyzed for homogeneity of dispersion by ANOVA. The parameters found to be significant in ANOVA were assessed by the Dunnett test. Significant differences (as shown in the plots) were classified as: * for $P<0.05$; more significant *: for $P<0.01$.

\section{Results}

\section{Effect of HZJW on $\mathrm{HCl} /$ Ethanol-induced gastric ulcer}

Ulcerative indices and gastroprotection percentage were determined in rats with $\mathrm{HCl} /$ Ethanol-induced ulcers by measuring ulcerative lesion length. As shown in Figure 1, intragastric administration of $\mathrm{HCl} /$ Ethanol to rats caused severe gastric mucosal damage (in the form of hemorrhagic streaks), while administration of HZJW displayed statistically significant anti-ulcerogenic activity, and dose-dependently reduced the ulcerative lesion index $(116.70 \pm 36.4 ; 102.20 \pm 18.20, P<0.05 ; 84.10 \pm 12.1$, $P<0.01$ at doses of 260,520 and $1040 \mathrm{mg} / \mathrm{kg}$, respectively), as compared with the model group $(134.10 \pm 31.69)$. Ranitidine also offered significant protection against $\mathrm{HCl} /$ Ethanol-induced gastric lesions $(20.40 \pm 6.93, \quad P<0.01)$. The percentages of ulcer inhibition were $12.98 \%, 23.79 \%, 37.29 \%$ and $45.04 \%$ for the groups treated with 260,520 and $1040 \mathrm{mg} / \mathrm{kg}$ of HZJW and ranitidine, respectively. 

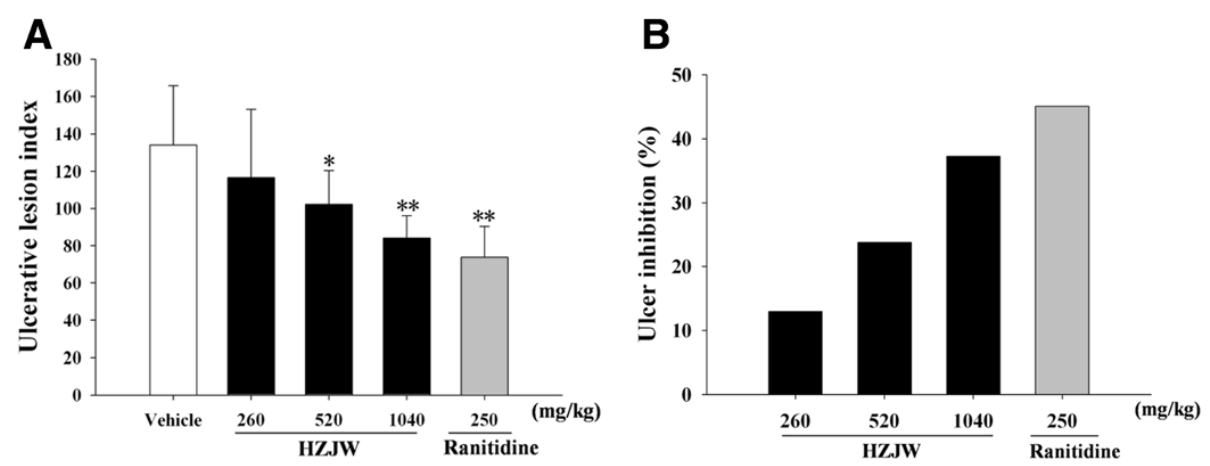

Figure 1 Protective effect of HZJW and raniditine on ulcerative lesion induced by $\mathrm{HCl} / \mathrm{Ethanol}$. (A) Ulcerative lesion index; (B) Ulcer inhibition (\%). Vehicle group (Open column), ranitidine group (250 mg/kg/d, grey bar), HZJW groups (260, 520, and 1040 mg/kg/d, dark bars). Vertical bars represent standard deviation (S.D.), where $n=10$. Asterisks designate significant differences: ${ }^{*} P<0.05 \&{ }^{* *} P<0.01$ vs. Vehicle group.

\section{Effect of HZJW on NSAID-induced ulcer}

To further verify the cytoprotective mechanism of HZJW against gastric ulcer, NSAIDs-induced mucosal damage model was employed. It was observed that aspirin produced extensive necrosis of the gastric mucosa, whereas animals treated with HZJW (260, 520 and $1040 \mathrm{mg} / \mathrm{kg}$ ) exhibited potent protection against aspirin-induced gastric ulceration in a dose-dependent manner. Orally applied HZJW significantly reduced the ulcer lesion index $(35.40 \pm 5.93 ; 31.30 \pm 8.08, P<0.05 ; 26.80 \pm 8.27$, $P<0.01)$ respectively, as compared to control group $(41.60 \pm 10.80)$. As well, ranitidine conferred prominent protection against ulcerogenesis $(20.40 \pm 6.93, P<0.01)$. The extent of inhibitions for the respective doses employed was $14.90,24.76,35.58$ and $50.96 \%$, respectively. These results were summarized in Figure 2.

\section{In vitro anti-H. pylori assay}

The agar dilution method has been standardized as the reference method for minimum inhibitory concentration (MIC) determinations by the Clinical And Laboratory
Standards Institute (CLSI). In the in vitro assay, MICs for HZJW and amoxicillin (positive control) were 125 and 0.12 $\mu \mathrm{g} / \mathrm{mL}$ respectively. The in vitro anti- $H$. pylori potency of amoxicillin was far more pronounced than that of HZJW.

\section{In vivo anti-H. pylori assay}

Taking into consideration these data as well as the limitations of in vitro efficacy experimentation, we also evaluated the in vivo eradication potency of HZJW in previously described rapid screening mouse model [20]. In the rapid urease assay, all animals in control group were negative for urease reaction, whereas in H. pylori-inoculated group 50\% were positive. Treatment with HZJW (520, 1040 and $2080 \mathrm{mg} / \mathrm{kg}$ ) increased the negativity of the urease test in a dose-related manner, reaching $83.33 \%(P<0.01), 76.5 \%$ and $72.2 \%$ $(P<0.05)$ respectively, in parallel to model animals. The test negativity rate was more prominent with HZJW treatment of lower dose. For the group given standard treatment--amoxicillin, negativity was $88.24 \%(P<0.01)$, as shown in Figure 3A.
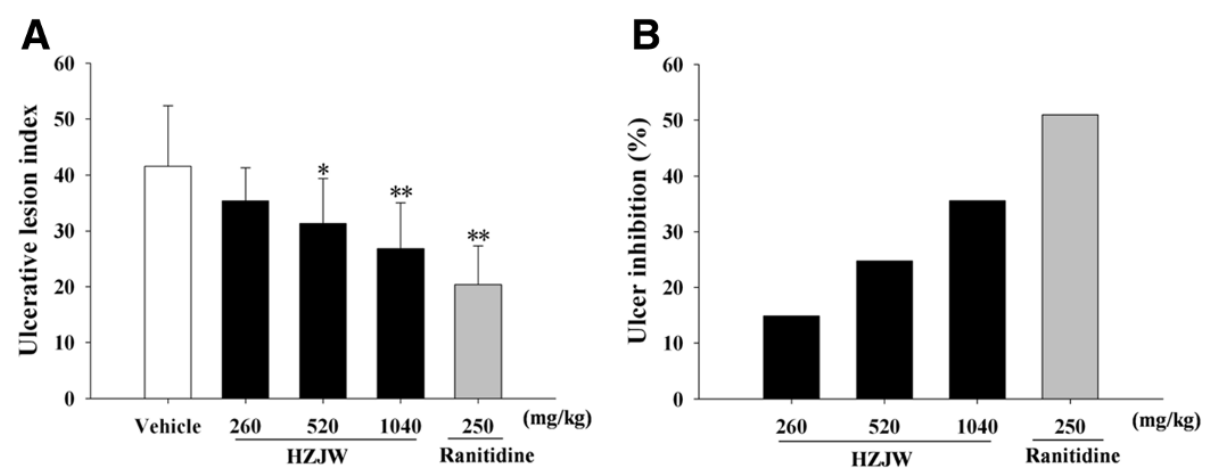

Figure 2 Protective effect of HZJW and raniditine on ulcerative lesion induced by NSAID. (A) Ulcerative lesion index; (B) Ulcer inhibition (\%). Vehicle group (Open column), ranitidine group (250 mg/kg/d, grey bar), HZJW groups (260, 520, and $1040 \mathrm{mg} / \mathrm{kg} / \mathrm{d}$, dark bars). Vertical bars represent standard deviation (S.D.), where $n=10$. Asterisks designate significant differences: ${ }^{*} P<0.05 \&{ }^{*} P<0.01$ vs. Vehicle group. 

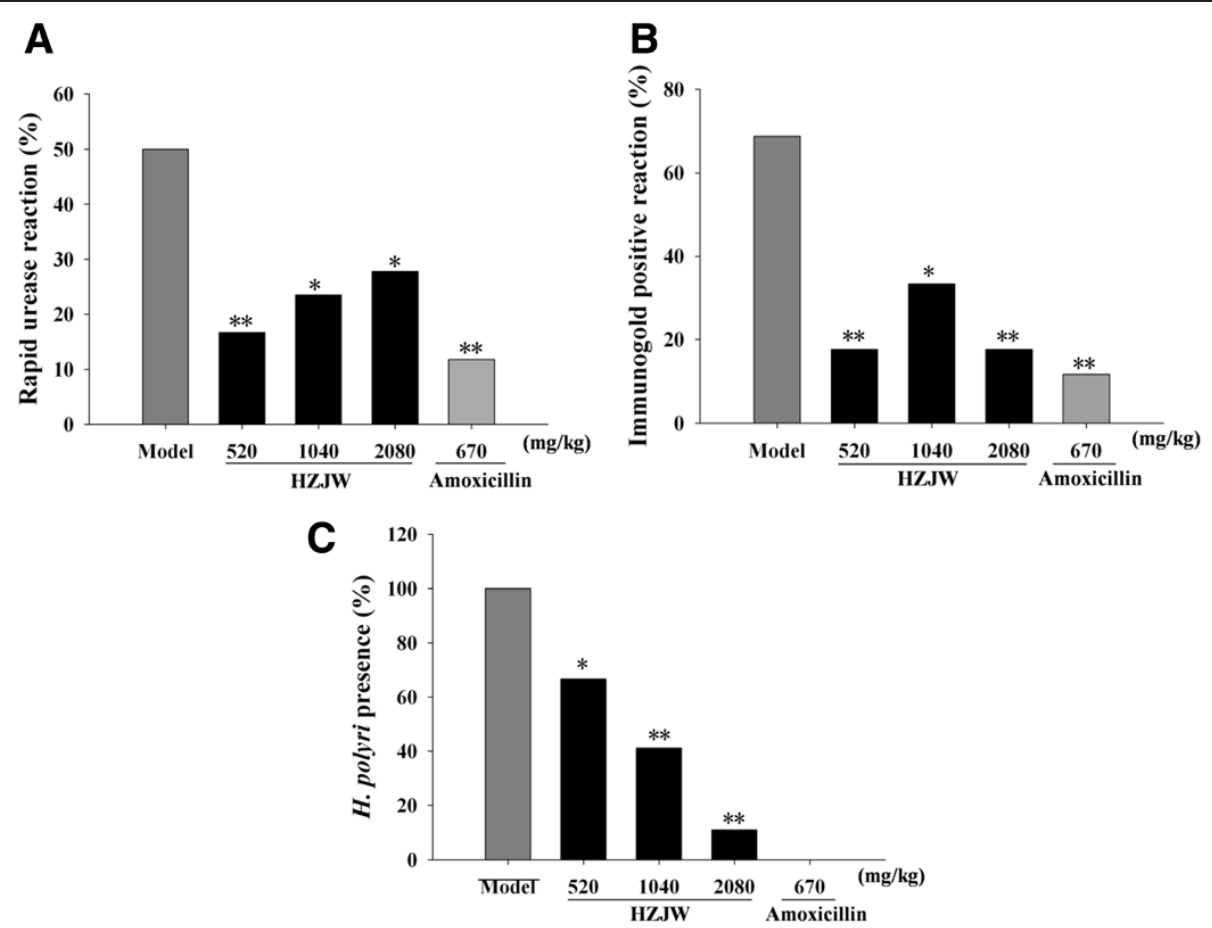

Figure 3 Effect of HZJW and amoxicillin on the eradication of Helicobacter pylori via rapid urease test (A), immunogold test (B) and histopathological analysis (C). Vehicle group (Open column), amoxicillin group (670 mg/kg/d, grey bar), HZJW groups (520, 1040 and $2080 \mathrm{mg} / \mathrm{kg} / \mathrm{d}$, dark bars). ${ }^{*} P<0.05 \&{ }^{* *} P<0.01$ vs. Vehicle group.

With respect to $H$. pylori urease Immunogold Test (Figure 3B), the infection rate for animals treated with HZJW of 520, 1040 and $2080 \mathrm{mg} / \mathrm{kg}$ was $17.65 \%$ $(P<0.01)$, 33.33\% $(P<0.05)$ and $17.65 \%(P<0.01)$ respectively, as compared with $68.75 \%$ of the model counterpart. The group treated with amoxicillin showed better results, in general, than those groups receiving HZJW with incidence rate of $11.76 \%(P<0.01)$.

In relation to the presence of $H$. pylori in gastric mucosa, HZJW treatment depleted the number of viable $H$. pylori in gastric tissues of inoculated animals. According to the data obtained (Figure $3 \mathrm{C}$ ), it was verified that those groups treated with 520, 1040 and $2080 \mathrm{mg} / \mathrm{kg}$ of HZJW presented a dose-dependent decrease in positive cases with carbolic acid \& basic fuchsin staining, by 66.67\% $(P<0.05), 41.18 \%(P<0.001)$ and $11.11 \%(P<0.01)$ respectively. Treatment with standard drugs caused complete elimination of $H$. pylori.

\section{Acute toxicity evaluation}

The acute dose study provides a guideline for selecting doses for the subacute and chronic low-dose study, which may be more clinically relevant [21]. In the acute toxicity study, the oral $\mathrm{LD}_{50}$ of HZJW was over $18.0 \mathrm{~g} / \mathrm{kg}$ by a single oral administration following fixed dose procedure. Animals did not manifest significant abnormal signs and alternations, behavioral changes, water or food consumption, body weight changes, or macroscopic findings at any time of observation. Autopsy results exhibited no significant change or lesion in the viscera of any animal.

\section{Survival and clinical signs}

In the chronic oral toxicity assessment,animals treated with doses of 5000,2500 and $1000 \mathrm{mg} / \mathrm{kg}$ (equivalent to 50-, 25- and 10-fold human clinical doses, respectively) was observed after 26 weeks of daily administration of HZJW, as well as after the 4-week recovery period. No drug-related deaths were observed in any of the HZJW-treated groups. Six rats of both sexes experienced slight fur loss and the region of hair loss was limited on grasping area including shoulder and back neck and just partial loss. The symptom disappeared after certain period. No other test-article-related clinical signs were observed in rats dosed up to $5000 \mathrm{mg} / \mathrm{kg}$ with HZJW. No abnormal findings were observed during the ophthalmological examination in any of the groups (data not shown).

\section{Body weights, organ weights and feed consumption}

In all groups, body weight gradually increased for 6 months, and changes in body weight in HZJW treated groups relative to the control group were not significant during the 
experimental period (Figure 4). As expected, the rats gained weight with time. From the 1st week to the 30th week, body weight of animals in three treatment group showed no significant differences compared with that of control. No statistically significant differences in food (Figure 5) consumption were detected between the control and treatment group regardless of sex or recovery group. Absolute and relative organ weights of 26-week treated rats are shown in Table 2. Since these changes in organ weight by the end of week 26 and recovery were not accompanied by any relevant histopathological change. The organ weight changes were not considered to be of toxicological significance by HZJW treatment.

\section{Hematological parameters}

Hematopoietic indices have been reported to be very sensitive to toxic compounds and serve as important index of physiologic and pathologic status for both animals and humans [22]. The effect of HZJW on the hematological parameters of the experimental and control rats is presented in Table 3. The results indicated that all hematological parameters measured remained within the physiological range throughout the 30-week experimental period. There were no significant changes in mean corpuscular volume, red cell distribution width, platelets, platelet distribution width, prothrombin time, eosinophils, basophils, monocytes, and reticulocytes values between the control and treated animals (main groups). Despite a significant change was observed in some parameters (hemoglobin, mean corpuscular hemoglobin, mean corpuscular hemoglobin concentration, mean platelet volume, white blood cell, lymphocytes, neutrophils), the above parameters remained within the range of normal physiological variation and there was no dose response relationship.

\section{Blood biochemistry}

The data for the biochemical parameters in the treated and control rats are presented in Table 4. All the parameters remained within the physiological range throughout the 30-week experimental period. No statistically significant differences were observed amongst the blood chemistry, including alkaline phosphatase activity, total cholesterol, urea, creatine phosphokinase activity, sodium ions, potassium ions and chlorideions between the control and treated animals. Despite some parameters experienced significant variation (glucose, triglycerides, aspartate aminotransferase activity, alanine aminotransferase activity, total protein, total bilirubin, direct bilirubin, alkaline phosphatase activity, albumin and urea), there were no dose response relationships and all of above parameters remained within the physiological range.

\section{Histopathogical findings}

Histopathological examinations are an important aspect of safety assessments. In the histopathological examination, no noteworthy HZJW related lesions were observed, though some abnormalities were found. The major pathological findings from the histopathological examination included minimal inflammatory cell foci and vacuolar degeneration in the liver, portal myocardial inflammatory cell infiltration in the heart, peri-bronchovascular chronic inflammation and, inflammatory cell foci hyperplasia in the lungs, the tubule interstitial chronic inflammation, tubule cortical vacuolar degeneration and protein cast in the kidneys. However, these symptoms are part of chronic progressive nephropathy often observed in rats of old age and is particularly frequently observed in males. These findings were noted in both sexes in a manner not dependent on dose. The high-dose group (5000 mg/kg) did not differ significantly from the control group in any organ. These changes were not considered to be treatment related,
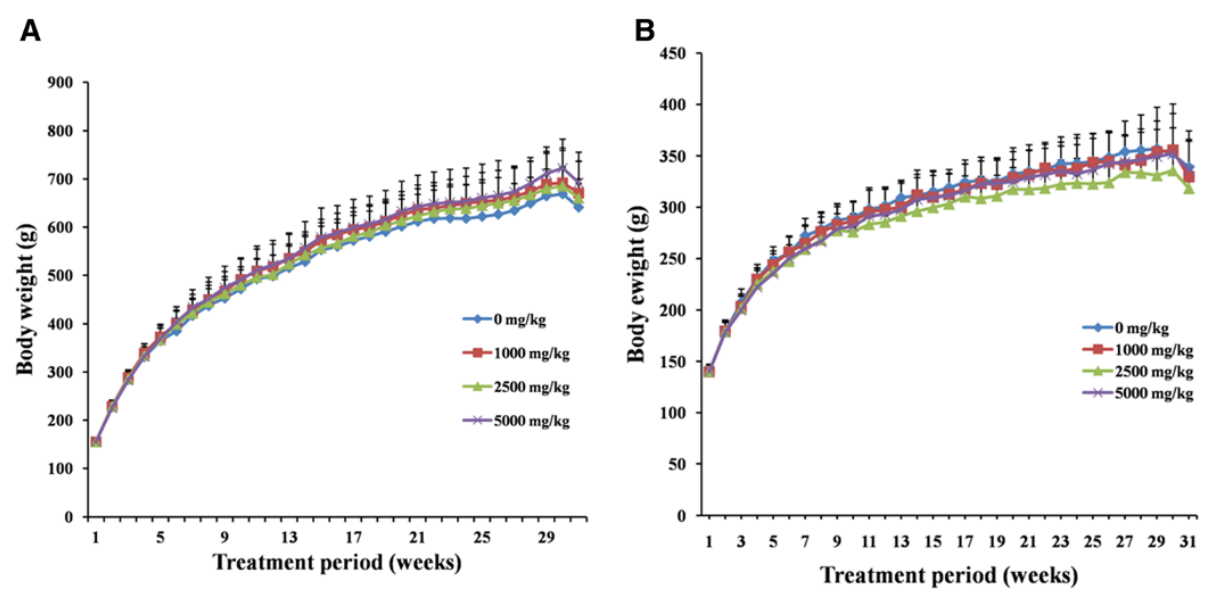

Figure 4 Mean body weight of male (A) and female (B) rats dosed with HZJW. 

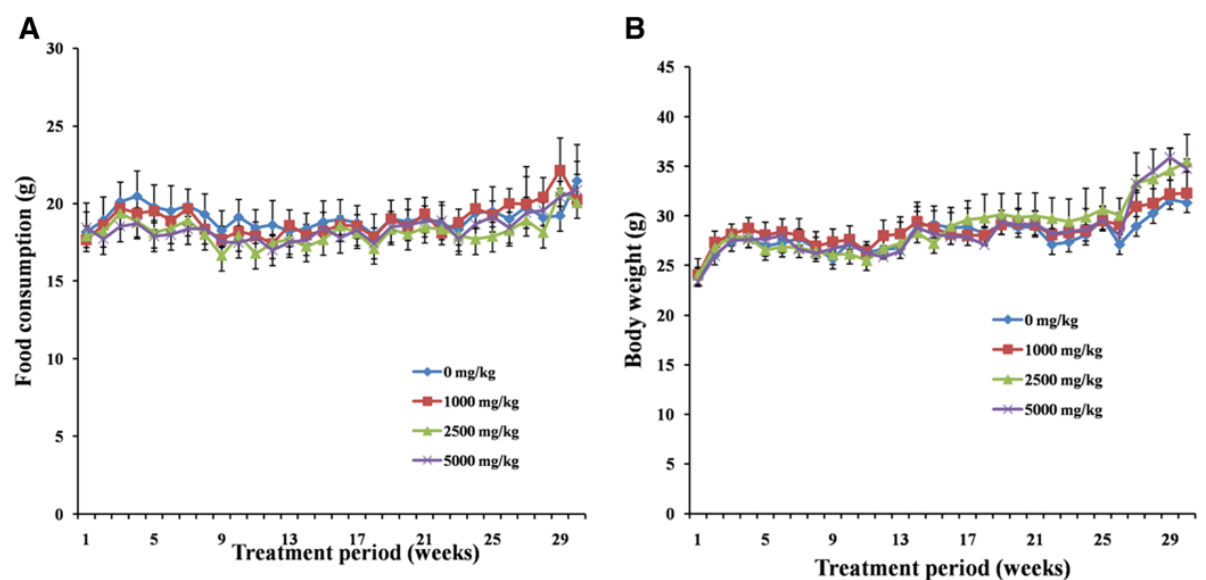

Figure 5 Mean food consumption by male (A) and female (B) rats treated with HZJW.

because these microscopic changes were commonly observed in untreated old rats.

\section{Discussion}

Currently, the commercial use of traditional herbal medicines has increased and various new drugs are being developed based on this development. Concerns have been raised over the lack of scientific evidence regarding the efficacy and safety of herbal products $[23,24]$, though some have been verified by clinical trials. Many researchers have undertaken studies to validate the efficacy of herbal prescriptions; however, few have initiated investigations addressing their safety and toxicity. Indeed, the toxicity of many of these herbal remedies has not been scientifically validated, and their safeties have been questioned recently due to reports of side effects and fatalities [25], hepatotoxicity [26], and nephrotoxicity [27]. Considering the complexity of general herbal prescriptions and their inherent biological variation, it is necessary to evaluate their safety, efficacy, and quality [28]. In this study, we examined the efficacy and safety of HZJW, an herbal-derived anti-ulcer formulation applied in clinics, via gastroprotective assessment, anti- $H$. pylori assay, oral acute toxicity and a systemic 6-month repeated-dose toxicity study.

The gastroprotective effect of HZJW was investigated by two animal models of acute gastric injury induced by necrotizing agents, i.e., $\mathrm{HCl} /$ Ethanol and NSAIDs. The $\mathrm{HCl} /$ Ethanol method is a rapid and convenient way of screening agents of antiulcer potency, which is assessed in terms of absence or reduction in macroscopically visible lesions [29]. $\mathrm{HCl} /$ Ethanol acts by exerting a direct toxic effect on the epithelium, inducing the formation of characteristic necrotic lesions due to a reduction in the mucus. Besides, it causes reduction of gastric blood flow, solubilization of mucus and bicarbonate secretion [30]. $\mathrm{HCl} /$ Ethanol induced gastric damage ranging from endothelial microvascular damage to development of macroscopic gastric mucosal lesions, which can be mainly attributed to the inhibition of cytoprotective prostaglandins (PG) biosynthesis [31]. A number of mechanisms that include enhanced gastric mucosal defense through increasing mucus and/or bicarbonate production, reducing gastric acid secretion or by simply neutralizing the gastric acidity [32], can mediate the gastric mucosal protection against $\mathrm{HCl} /$ Ethanol. In the present study, the control group subjected to $\mathrm{HCl} /$ Ethanol clearly produced the expected characteristic zone of necrotizing mucosal lesions, while pretreatment with HZJW had significantly and dose-dependently decreased the ulcerative index and the percentage of lesion, thus markedly improved ulcer healing. These results indicated that HZJW exhibited protective effect against $\mathrm{HCl}$ /Ethanol-induced ulcerogenesis in rats.

Another experimental protocol employed in the investigation was NSAIDs- induced ulcer by aspirin, which induced gastric lesions due to the distinct mechanism in generating ulcer lesions compared with $\mathrm{HCl} /$ Ethanol. NSAIDs induce injury/bleeding via three key pathways: inhibition of cyclooxygenase (COX)-1 activity, inhibition of COX-2 activity, and direct cytotoxic effects on the epithelium [33]. The main importance of the systemic effects of NSAIDs, in terms of inducing gastric ulceration, is their ability to suppress prostaglandin synthesis [34]. In the stomach, prostaglandins play an important protective role by stimulating the secretion of bicarbonate and mucus, maintaining the blood flow of the mucosa, and they are responsible for regulating mucosal cell renewal [35]. Aspirin is a cyclooxygenase inhibitor which suppresses gastroduodenal bicarbonate secretion, reduces endogeneous prostaglandin biosynthesis and disrupts the mucosal barrier as well as mucosal blood flow in animals, causing increased susceptibility to gastric mucosal lesions [36]. In 


\begin{tabular}{|c|c|c|c|c|c|c|c|c|c|c|c|c|c|}
\hline \multirow{3}{*}{ Parameter } & \multirow{3}{*}{ Sex } & \multicolumn{12}{|c|}{ Dose (mg/kg) } \\
\hline & & \multicolumn{3}{|c|}{$0 \mathrm{mg} / \mathrm{kg}$} & \multicolumn{3}{|c|}{$1000 \mathrm{mg} / \mathrm{kg}$} & \multicolumn{3}{|c|}{$2500 \mathrm{mg} / \mathrm{kg}$} & \multicolumn{3}{|c|}{$5000 \mathrm{mg} / \mathrm{kg}$} \\
\hline & & $13 \mathrm{wk}$ & 26 wk & covery & 13 wk & $26 \mathrm{wk}$ & Recovery & $13 \mathrm{wk}$ & $26 \mathrm{wk}$ & Reco & $13 w k$ & 26 wk & Recovery \\
\hline \multirow{2}{*}{$\begin{array}{c}\text { Body } \\
\text { weight }\end{array}$} & Male & $36.2 \pm 16.4$ & $4.4 \pm 70.0$ & $641.3 \pm 95.7$ & $516.3 \pm 101.3$ & $634.6 \pm 60.1$ & $670.7 \pm 65.7$ & $482.3 \pm 44.4$ & $628.1 \pm 67.7$ & $660.4 \pm 39.7$ & $481.8 \pm 39.5$ & $637.8 \pm 82.5$ & $691.7 \pm 63.8$ \\
\hline & Female & $291.3 \pm 16.8$ & $331.8 \pm 27.2$ & $338.9 \pm 35.6$ & $280.9 \pm 17.0$ & $333.4 \pm 32.2$ & $329.7 \pm 34.7$ & $266.0 \pm 9.3$ & $308.3 \pm 25.7$ & $318.4 \pm 15.2$ & $269.0 \pm 36.4$ & $322.2 \pm 31.9$ & $336.6 \pm 29.5$ \\
\hline \multirow[t]{2}{*}{ Brain } & Male & $.096 \pm 0.064$ & $2.240 \pm 0.124$ & $2.163 \pm 0.103$ & $2.214 \pm 0.115$ & $2.221 \pm 0.095$ & $2.191 \pm 0.090$ & $2.080 \pm 0.117$ & $2.281 \pm 0.079$ & $2.224 \pm 0.092$ & $2.092 \pm 0.183$ & $2.238 \pm 0.089$ & $2.261 \pm 0.049$ \\
\hline & 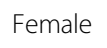 & $206+0066$ & \pm 0.064 & $38 \pm 0.096$ & $77 \pm 0.040$ & \pm 0.064 & 2 & 46 & 2 & 3 & 1 & 060 & 39 \\
\hline \multirow{2}{*}{$\begin{array}{c}\% \text { to body } \\
\text { weight }\end{array}$} & M & $0.431 \pm .016$ & 4 & 5 & 67 & 3 & 4 & 39 & 6 & 27 & 0 & 46 & 30 \\
\hline & Female & $0.687 \pm 0.041$ & $0.612 \pm 0.041$ & $0.621 \pm 0.066$ & $0.695 \pm 0.045$ & $0.608 \pm 0.060$ & $0.620 \pm 0.065$ & $0.754 \pm 0.023^{*}$ & $0.655 \pm 0.057$ & $0.630 \pm 0.019$ & $0.747 \pm 0.082$ & $0.638 \pm 0.065$ & $0.606 \pm 0.051$ \\
\hline \multirow[t]{2}{*}{ Thymus } & Male & $0.297 \pm 0.097$ & $0.270 \pm 0.069$ & $0.262 \pm 0.063$ & $0.351 \pm 0.170$ & $0.219 \pm 0.106$ & $0.250 \pm 0.092$ & $0.313 \pm 0.043$ & $0.223 \pm 0.084$ & $0.200 \pm 0.038$ & $0.270 \pm 0.048$ & $0.262 \pm 0.056$ & $0.224 \pm 0.059$ \\
\hline & Female & $0.367 \pm 0.057$ & 73 & 57 & .041 & 38 & $0.225 \pm 0.045$ & 0.2 & 0.226 & 0.2 & 0.091 & 0.231 & 023 \\
\hline \multirow{2}{*}{$\begin{array}{c}\% \text { to body } \\
\text { weight }\end{array}$} & Male & $61 \pm 0.018$ & $044 \pm 0.009$ & $0.041 \pm 0.008$ & $0.065 \pm 0.019$ & $0.034 \pm 0.013^{*}$ & $0.037 \pm 0.013$ & $0.065 \pm 0.011$ & $0.035 \pm 0.012$ & $0.030 \pm 0.005^{*}$ & $0.056 \pm 0.008$ & $0.041 \pm 0.008$ & .006 \\
\hline & Female & 17 & 23 & 4 & 5 & 0 & 5 & 8 & 0 & 0. & 0. & 0. & 9 \\
\hline \multirow[t]{2}{*}{ Heart } & Male & $381 \pm 0.111$ & $1.625 \pm 0.204$ & $1.653 \pm 0.259$ & $1.494 \pm 0.193$ & $1.710 \pm 0.229$ & $1.613 \pm 0.225$ & $1.408 \pm 0.156$ & $1.702 \pm 0.169$ & $1.749 \pm 0.138$ & $1.467 \pm 0.093$ & $1.699 \pm 0.182$ & $1.912=$ \\
\hline & Female & $0.832 \pm 0.042$ & $1.100 \pm 0.101$ & $1.046 \pm 0.138$ & $0.917 \pm 0.094$ & $1.089 \pm 0.109$ & $1.013 \pm 0.091$ & $0.905 \pm 0.066$ & $1.024 \pm 0.091$ & $0.986 \pm 0.069$ & $0.969 \pm 0.102$ & $1.098 \pm 0.136$ & $1.014 \pm 0.068$ \\
\hline \multirow{2}{*}{$\begin{array}{l}\% \text { to body } \\
\text { weight }\end{array}$} & Male & $284+0014$ & $0.270 \pm 0.029$ & $0.258 \pm 0.004$ & $0.294 \pm 0.037$ & $0.271 \pm 0.039$ & $0.240 \pm 0.015^{*}$ & $0.292 \pm 0.021$ & $0.272 \pm 0.021$ & $0.265 \pm 0.020$ & $0.306 \pm 0.028$ & $0.268 \pm 0.016$ & $0.277 \pm 0.016$ \\
\hline & Female & $0.286 \pm 0.012$ & $0.333 \pm 0.034$ & $0.308 \pm 0.010$ & $0.326 \pm 0.023^{*}$ & $0.328 \pm 0.027$ & $0.308 \pm 0.016$ & $0.341 \pm 0.036^{*}$ & $0.334 \pm 0.044$ & $0.070-$ & $0.362 \pm 0.025^{* *}$ & $0.341 \pm 0.032$ & $0.302 \pm 0.018$ \\
\hline \multirow[t]{2}{*}{ Lung } & Male & 01 & $1.831=$ & $1.845 \pm 0.237$ & $1.862 \pm 0.779$ & $1.876 \pm 0.246$ & $1.910 \pm 0.094$ & $1.528 \pm 0.103^{*}$ & $1.930 \pm 0.088$ & $1.916 \pm 0.052$ & $1.516 \pm 0.066^{*}$ & $1.898 \pm 0.127$ & 2.099 \\
\hline & Female & $1.210 \pm 0.061$ & $1.434 \pm 0.110$ & $1.409 \pm 0.098$ & $1.506 \pm 0.538$ & $1.449 \pm 0.168$ & $1.28 \pm 0.062^{*}$ & $1.210 \pm 0.072$ & $1.438 \pm 0.166$ & $1.419 \pm 0.048$ & $1.204 \pm 0.192$ & $1.443 \pm 0.138$ & $1.503 \pm 0.152$ \\
\hline \multirow{2}{*}{$\begin{array}{c}\% \text { to body } \\
\text { weight }\end{array}$} & Male & $0.366 \pm 0.038$ & $0.304 \pm 0.041$ & $0.289 \pm 0.025$ & $0.354 \pm 0.087$ & $0.296 \pm 0.034$ & $0.287 \pm 0.033$ & $0.318 \pm 0.021^{*}$ & $0.310 \pm 0.029$ & $0.291 \pm 0.016$ & $0.316 \pm 0.026$ & $0.300 \pm 0.028$ & $0.303 \pm 0.035$ \\
\hline & Female & $0.416 \pm 0.024$ & $0.434 \pm 0.036$ & $0.418 \pm 0.043$ & $0.537 \pm 0.189$ & $0.438 \pm 0.064$ & $0.394 \pm 0.041$ & $0.456 \pm 0.037$ & $0.466 \pm 0.036$ & $0.446 \pm 0.025$ & $0.447 \pm 0.021$ & $0.450 \pm 0.048$ & 0.448 \\
\hline \multirow[t]{2}{*}{ Liver } & Male & $.492 \pm 1.894$ & $12.852 \pm 1.753$ & $14.019 \pm 2.841$ & $12.564 \pm 3.570$ & 3 & 9 & $11.882 \pm 1.267$ & $14.860 \pm 2.256^{*}$ & $15.212 \pm 1.286$ & $11.380 \pm 0.892$ & $16.043 \pm 3.106^{*}$ & 16.3 \\
\hline & Female & $6.885 \pm 0.495$ & 4 & 2 & 19 & 34 & 18 & 05 & 8. & 94 & 7.3 & 8.7 & 1.5 \\
\hline & Male & 2 & $2.125 \pm 0.125$ & 2.180 & $2.407 \pm 0.256$ & $2.117 \pm 0.187$ & $2.084 \pm 0.143$ & $2.461 \pm 0.062$ & $2.363 \pm 0.209^{* *}$ & $2.301 \pm 0.061$ & $2.366 \pm 0.123$ & $2.498 \pm 0.193^{* *}$ & $2.357 \pm 0.164$ \\
\hline & Female & $2.369 \pm 0.223$ & $2.481 \pm 0.355$ & $2.239 \pm 0.151$ & $2.510 \pm 0.189$ & $2.561 \pm 0.237$ & $2.351 \pm 0.132$ & $2.605 \pm 0.169$ & $2.644 \pm 0.205$ & $2.402 \pm 0.171$ & $2.728 \pm 0.105^{*}$ & $2.728 \pm 0.300$ & $2.258 \pm 0.159$ \\
\hline \multirow[t]{2}{*}{ Spleen } & Male & $0.614 \pm 0.042$ & $0.799 \pm 0.116$ & $0.833 \pm 0.115$ & $0.639 \pm 0.105$ & $0.849 \pm 0.110$ & $0.795 \pm 0.096$ & $0.708 \pm 0.166$ & $0.810 \pm 0.115$ & $0.868 \pm 0.133$ & $0.673 \pm 0.073$ & $0.916 \pm 0.101^{*}$ & $0.859 \pm 0.104$ \\
\hline & e & $431 \pm 0.023$ & $0.618 \pm 0.149$ & $82 \pm 0.108$ & $50 \pm 0.054$ & $44 \pm 0.074$ & $0.553 \pm 0.0$ & $0.478 \pm 0.035$ & $0.574 \pm 0$ & $0515+0$ & $0.446 \pm 0$ & 0.5 & 7 \\
\hline \multirow{2}{*}{$\begin{array}{c}\% \text { to body } \\
\text { weight }\end{array}$} & ale & 006 & $0.133 \pm 0.022$ & $0.130 \pm 0.009$ & $0.126 \pm 0.021$ & $0.134 \pm 0.009$ & $0.118 \pm 0.007$ & $0.147 \pm 0.030$ & $0.129 \pm 0.009$ & $1 \pm 0.018$ & $0.140 \pm 0.015$ & $0.145 \pm 0.015$ & $0.124 \pm 0.009$ \\
\hline & Female & $0.148 \pm 0.009$ & $0.187 \pm 0.046$ & $0.172 \pm 0.026$ & $0.164 \pm 0.020$ & $0.164 \pm 0.021$ & $0.168 \pm 0.013$ & $0.180 \pm 0.016^{* *}$ & $0.186 \pm 0.033$ & $0.171 \pm 0.006$ & $0.167 \pm 0.028$ & $0.162 \pm 0.026$ & $0.162 \pm 0.018$ \\
\hline \multirow[t]{2}{*}{ Kidney } & Male & $2.832 \pm 0.228$ & $3.343 \pm 0.322$ & $3.406 \pm 0.309$ & $2.943 \pm 0.433$ & $3.421 \pm 0.481$ & $3.449 \pm 0.451$ & $2.766 \pm 0.141$ & $3.519 \pm 0.316$ & $3.856 \pm 0.456$ & $3.013 \pm 0.428$ & $3.622 \pm 0.410$ & $4.178 \pm 0.503^{*}$ \\
\hline & Fen & 1.65 & 1.8 & 1. & 2 & 5 & 5 & 45 & 5 & 1 & 1 & 54 & 1.8 \\
\hline \multirow{2}{*}{$\begin{array}{c}\% \text { to body } \\
\text { weight }\end{array}$} & Male & $582 \pm 0$ & 56 & 50 & 70 & 10 & 29 & $0.577 \pm 0$ & $0.563 \pm$ & 52 & 0.62 & 59 & 0.026 \\
\hline & Female & $0.569 \pm 0.052$ & $0.574 \pm 0.058$ & $0.552 \pm 0.031$ & $0.558 \pm 0.048$ & $0.582 \pm 0.054$ & $0.581 \pm 0.049$ & $0.604 \pm 0.027$ & $0.643 \pm 0.049^{* *}$ & $0.594 \pm 0.024^{*}$ & $0.606 \pm 0.029^{*}$ & $0.632 \pm 0.056$ & $0.552 \pm 0.034$ \\
\hline
\end{tabular}


Table 2 Absolute \& relative organ weights in rats orally treated with HZJW (Continued)

\begin{tabular}{|c|c|c|c|c|c|c|c|c|c|c|c|c|c|}
\hline \multirow{2}{*}{$\begin{array}{l}\text { Adrenal } \\
\text { grand }\end{array}$} & le & 2 & 6 & 9 & 013 & 7 & $0.051 \pm$ & 12 & 9 & 5 & $0.053 \pm 0.009^{*}$ & 8 & 0 \\
\hline & male & 7 & 9 & 9 & 9 & & * & 6 & & 11 & & 9 & \\
\hline \multirow{2}{*}{$\begin{array}{l}\% \text { to body } \\
\text { weight }\end{array}$} & le & $14 \pm 0.002$ & 2 & 1 & 4 & 01 & * & 03 & 2 & 001 & 22 & 002 & 001 \\
\hline & male & c & 33 & 2 & 3 & 3 & * & 03 & 4 & 44 & 4 & 3 & 03 \\
\hline \multirow{2}{*}{$\begin{array}{l}\text { Testis(Rt.)/ } \\
\text { Ovary (Lt.) }\end{array}$} & Male & $391 \pm 0.243$ & 8 & 6 & 2 & 8 & 5 & 89 & 2 & & & 51 & \\
\hline & male & 2 & 30 & 49 & 17 & 38 & 77 & 17 & 2 & 6 & & & \\
\hline \multirow{2}{*}{$\begin{array}{c}\% \text { to body } \\
\text { weight }\end{array}$} & ale & 52 & 054 & 050 & 109 & 99 & 33 & 95 & 779 & 2 & 14 & 8 & 46 \\
\hline & Female & - & 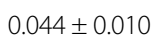 & 4 & * & 3 & 5 & * & $0.051 \pm 0.006$ & 6 & 2 & & 6 \\
\hline \multirow{2}{*}{$\begin{array}{l}\text { Epididymis } \\
\text { (Lt.)/Uterus }\end{array}$} & te & $1.264 \pm 0.099$ & 05 & 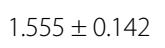 & 7 & 9 & 3 & 0 & o & & $80^{*}$ & 1 & 52 \\
\hline & Female & $0.649 \pm$ & 289 & 114 & 283 & 340 & 0. & 195 & 13 & 52 & 338 & 0. & .365 \\
\hline \multirow{2}{*}{$\begin{array}{l}\% \text { to body } \\
\text { weight }\end{array}$} & Male & $0.260 \pm 0.021$ & 4 & 6 & 0 & 7 & 3 & 5 & 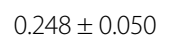 & 2 & 35 & 3 & 29 \\
\hline & Female & $0.225 \pm 0.070$ & $0.245 \pm 0.092$ & $0.264 \pm 0.048$ & $0.315 \pm 0.108$ & $0.299 \pm 0.102$ & $0.296 \pm 0.084$ & $0.269 \pm 0.074$ & $0.298 \pm 0.082$ & $0.217 \pm 0.044$ & $0.272 \pm 0.084$ & $0.240 \pm 0.082$ & $0.271 \pm 0.125$ \\
\hline
\end{tabular}

Note: Data represents body weights right before necropsy, after fasting; Values are mean \pm S.D. ( $n=5$ (4 weeks or recovery), 10 (26 weeks), recovery (30 weeks)/sex/dose).

Note: Data represents body weights right before necrop
${ }^{*} P 0.05 \&$ \& $P<0.01$ compared with normal group. 
Table 3 Hematological values in rats orally treated with HZJW

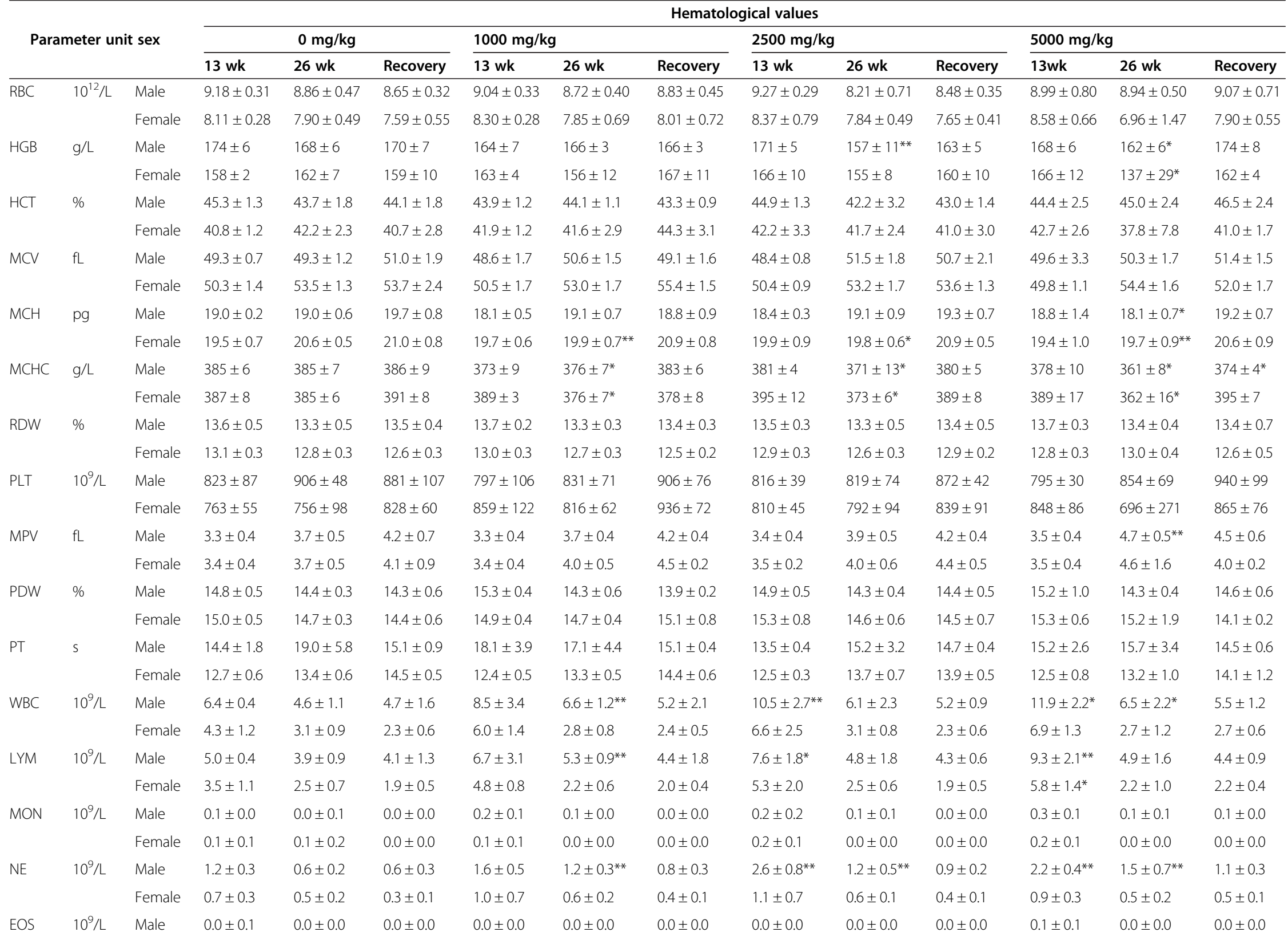


Table 3 Hematological values in rats orally treated with HZJW (Continued)

\begin{tabular}{|c|c|c|c|c|c|c|c|c|c|c|c|c|c|c|}
\hline & & Female & $0.0 \pm 0.1$ & $0.0 \pm 0.0$ & $0.0 \pm 0.0$ & $0.1 \pm 0.0$ & $0.0 \pm 0.0$ & $0.0 \pm 0.0$ & $0.0 \pm 0.1$ & $0.0 \pm 0.0$ & $0.0 \pm 0.0$ & $0.0 \pm 0.1$ & $0.0 \pm 0.0$ & $0.0 \pm 0.0$ \\
\hline \multirow[t]{2}{*}{ BAS } & $10^{9} / \mathrm{L}$ & Male & $0.0 \pm 0.0$ & $0.0 \pm 0.0$ & $0.0 \pm 0.0$ & $0.0 \pm 0.0$ & $0.0 \pm 0.0$ & $0.0 \pm 0.0$ & $0.0 \pm 0.0$ & $0.0 \pm 0.0$ & $0.0 \pm 0.0$ & $0.0 \pm 0.0$ & $0.0 \pm 0.0$ & $0.0 \pm 0.0$ \\
\hline & & Female & $0.0 \pm 0.0$ & $0.0 \pm 0.0$ & $0.0 \pm 0.0$ & $0.0 \pm 0.0$ & $0.0 \pm 0.0$ & $0.0 \pm 0.0$ & $0.0 \pm 0.0$ & $0.0 \pm 0.0$ & $0.0 \pm 0.0$ & $0.0 \pm 0.0$ & $0.0 \pm 0.0$ & $0.0 \pm 0.0$ \\
\hline \multirow[t]{2}{*}{ LYM } & $\%$ & Male & $77.7 \pm 4.5$ & $84.9 \pm 0.9$ & $86.7 \pm 1.0$ & $77.5 \pm 5.2$ & $80.7 \pm 2.7^{* *}$ & $83.4 \pm 0.6^{* *}$ & $72.8 \pm 4.0$ & $77.3 \pm 2.4^{*}$ & $80.8 \pm 1.8^{* *}$ & $78.0 \pm 4.7$ & $75.9 \pm 3.6^{* *}$ & $79.0 \pm 3.1^{* *}$ \\
\hline & & Female & $80.2 \pm 6.1$ & $80.0 \pm 2.1$ & $85.7 \pm 0.7$ & $80.3 \pm 7.7$ & $77.5 \pm 3.7$ & $83.9 \pm 1.4^{*}$ & $79.8 \pm 7.8$ & $77.2 \pm 2.3^{*}$ & $81.7 \pm 2.6^{*}$ & $81.7 \pm 6.2$ & $78.9 \pm 4.9$ & $80.0 \pm 2.0^{* *}$ \\
\hline \multirow[t]{2}{*}{ MON } & $\%$ & Male & $1.8 \pm 0.3$ & $1.1 \pm 0.5$ & $0.4 \pm 0.1$ & $1.9 \pm 1.1$ & $1.1 \pm 0.4$ & $0.5 \pm 0.3$ & $2.3 \pm 0.7$ & $1.4 \pm 0.7$ & $0.5 \pm 0.2$ & $2.7 \pm 0.6$ & $1.3 \pm 0.5$ & $1.1 \pm 0.4$ \\
\hline & & Female & $1.5 \pm 0.5$ & $2.4 \pm 4.3$ & $0.3 \pm 0.2$ & $2.6 \pm 0.5$ & $0.9 \pm 0.4$ & $0.3 \pm 0.3$ & $2.7 \pm 1.1$ & $1.2 \pm 0.6$ & $0.6 \pm 0.4$ & $3.0 \pm 0.6$ & $0.9 \pm 0.6$ & $0.5 \pm 0.3$ \\
\hline \multirow[t]{2}{*}{ NE } & $\%$ & Male & $19.5 \pm 4.8$ & $13.4 \pm 0.7$ & $12.3 \pm 1.1$ & $20.0 \pm 4.8$ & $17.6 \pm 3.0^{* *}$ & $15.8 \pm 0.6$ & $24.5 \pm 4.0$ & $20.6 \pm 2.6^{*}$ & $18.1 \pm 1.7$ & $18.7 \pm 4.3$ & $22.0 \pm 3.6^{* *}$ & $19.3 \pm 3.4$ \\
\hline & & Female & $16.8 \pm 6.3$ & $16.9 \pm 4.4$ & $13.8 \pm 0.7$ & $15.5 \pm 7.9$ & $20.7 \pm 3.5$ & $15.2 \pm 1.5$ & $16.6 \pm 8.7$ & $20.6 \pm 2.7^{*}$ & $16.7 \pm 2.6$ & $14.2 \pm 6.0$ & $19.3 \pm 4.1$ & $18.7 \pm 1.8$ \\
\hline \multirow[t]{2}{*}{ EOS } & $\%$ & Male & $0.7 \pm 0.3$ & $0.4 \pm 0.3$ & $0.4 \pm 0.40$ & $0.4 \pm 0.3$ & $0.3 \pm 0.2$ & $0.2 \pm 0.1$ & $0.3 \pm 0.2$ & $0.4 \pm 0.3$ & $0.4 \pm 0.3$ & $0.5 \pm 0.2$ & $0.5 \pm 0.4$ & $0.4 \pm 0.2$ \\
\hline & & Female & $1.2 \pm 0.2$ & $0.5 \pm 0.2$ & $0.1 \pm 0.1$ & $1.0 \pm 0.3$ & $0.5 \pm 0.2$ & $0.5 \pm 0.1$ & $0.7 \pm 0.5$ & $0.7 \pm 0.5$ & $0.8 \pm 0.6$ & $0.8 \pm 0.4$ & $0.8 \pm 0.5$ & $0.5 \pm 0.4$ \\
\hline \multirow[t]{2}{*}{ BAS } & $\%$ & Male & $0.3 \pm 0.1$ & $0.3 \pm 0.2$ & $0.2 \pm 0.1$ & $0.2 \pm 0.2$ & $0.3 \pm 0.2$ & $0.2 \pm 0.1$ & $0.1 \pm 0.1$ & $0.3 \pm 0.2$ & $0.1 \pm 0.1$ & $0.1 \pm 0.1$ & $0.3 \pm 0.1$ & $0.2 \pm 0.0$ \\
\hline & & Female & $0.2 \pm 0.2$ & $0.3 \pm 0.3$ & $0.1 \pm 0.1$ & $0.5 \pm 0.2$ & $0.4 \pm 0.3$ & $0.1 \pm 0.1$ & $0.2 \pm 0.3$ & $0.2 \pm 0.2$ & $0.2 \pm 0.3$ & $0.3 \pm 0.1$ & $0.1 \pm 0.2$ & $0.2 \pm 0.3$ \\
\hline \multirow[t]{2}{*}{ RET } & $\%$ & Male & $11 \pm 4$ & $21 \pm 5$ & $10 \pm 3$ & $14 \pm 6$ & $18 \pm 3$ & $12 \pm 2$ & $16 \pm 3^{*}$ & $18 \pm 7$ & $11 \pm 4$ & $14 \pm 4$ & $21 \pm 5$ & $11 \pm 4$ \\
\hline & & Female & $18 \pm 4$ & $21 \pm 4$ & $10 \pm 3$ & $19 \pm 5$ & $22 \pm 4$ & $17 \pm 2$ & $18 \pm 4$ & $18 \pm 6$ & $11 \pm 3$ & $14 \pm 4$ & $28 \pm 7$ & $10 \pm 4$ \\
\hline
\end{tabular}

Note: Red blood cell (RBC), hemoglobin (HGB), hematocrit (HCT), mean corpuscular volume (MCV), mean corpuscular HGB (MCH), mean corpuscular HGB concentration (MCHC), red cell distribution width (RDW),

basophils (BAS) prothrombin time (PT). Values are mean \pm S.D. $\left(n=5\right.$ (4 weeks), 10 (26 weeks) or recovery (30 weeks)/sex/dose). ${ }^{*} P<0.05 \& * * P<0.01$ compared with normal group. 


\begin{tabular}{|c|c|c|c|c|c|c|c|c|c|c|c|c|c|c|}
\hline \multirow{3}{*}{\multicolumn{3}{|c|}{ Parameter unit sex }} & \multicolumn{12}{|c|}{ Dose $(\mathrm{mg} / \mathrm{kg})$} \\
\hline & & & \multicolumn{3}{|c|}{$0 \mathrm{mg} / \mathrm{kg}$} & \multicolumn{3}{|c|}{$1000 \mathrm{mg} / \mathrm{kg}$} & \multicolumn{3}{|c|}{$2500 \mathrm{mg} / \mathrm{kg}$} & \multicolumn{3}{|c|}{$5000 \mathrm{mg} / \mathrm{kg}$} \\
\hline & & & $13 \mathrm{wk}$ & $26 w k$ & Recovery & $13 \mathrm{wk}$ & $26 \mathrm{wk}$ & Recovery & $13 \mathrm{wk}$ & $26 w k$ & Recovery & $13 w k$ & $26 \mathrm{wk}$ & Recovery \\
\hline \multirow[t]{2}{*}{$\overline{\mathrm{ALT}}$} & $U / L$ & Male & $40 \pm 3$ & $39 \pm 11$ & $34 \pm 8$ & $31 \pm 8$ & $42 \pm 11$ & $34 \pm 12$ & $41 \pm 4$ & $34 \pm 10$ & $47 \pm 14$ & $41 \pm 10$ & $30 \pm 6$ & $37 \pm 9$ \\
\hline & & Female & $43 \pm 8$ & $44 \pm 22$ & $32 \pm 8$ & $29 \pm 8^{*}$ & $59 \pm 32$ & $39 \pm 26$ & $33 \pm 9$ & $39 \pm 23$ & $41 \pm 43$ & $27 \pm 4^{* *}$ & $28 \pm 6$ & $28 \pm 7$ \\
\hline \multirow[t]{2}{*}{ AST } & $U / L$ & Male & $161 \pm 6$ & $109 \pm 28$ & $80 \pm 15$ & $114 \pm 24^{* *}$ & $113 \pm 36$ & $91 \pm 40$ & $124 \pm 13^{* *}$ & $83 \pm 13$ & $112 \pm 21$ & $121 \pm 20^{* *}$ & $87 \pm 25$ & $105 \pm 21$ \\
\hline & & Female & $161 \pm 35$ & $122 \pm 81$ & $78 \pm 17$ & $113 \pm 18^{*}$ & $155 \pm 72$ & $100 \pm 42$ & $127 \pm 27$ & $104 \pm 45$ & $86 \pm 71$ & $98 \pm 14^{* *}$ & $81 \pm 20$ & $65 \pm 12$ \\
\hline \multirow[t]{2}{*}{$L P$} & $U / L$ & Male & $90 \pm 10$ & $106 \pm 38$ & $77 \pm 27$ & $105 \pm 29$ & $70 \pm 15$ & $80 \pm 43$ & $106 \pm 27$ & $79 \pm 21$ & $84 \pm 14$ & $98 \pm 22$ & $68 \pm 20$ & $93 \pm 15$ \\
\hline & & Female & $61 \pm 19$ & $51 \pm 15$ & $59 \pm 17$ & $60 \pm 11$ & $46 \pm 18$ & $48 \pm 17$ & $55 \pm 23$ & $54 \pm 11$ & $41 \pm 22$ & $55 \pm 23$ & $44 \pm 15$ & $57 \pm 19$ \\
\hline \multirow[t]{2}{*}{ GLU } & $\mathrm{mmol} / \mathrm{L}$ & Male & $7.26 \pm 0.73$ & $6.07 \pm 0.78$ & $7.89 \pm 1.27$ & $6.99 \pm 1.49$ & $6.69 \pm 0.75$ & $7.85 \pm 1.03$ & $6.27 \pm 0.68$ & $7.84 \pm 1.30$ & $7.48 \pm 0.84$ & $7.38 \pm 0.78$ & $8.39 \pm 0.78^{* *}$ & $8.55 \pm 1.22$ \\
\hline & & Female & $7.60 \pm 0.95$ & $6.57 \pm 0.80$ & $7.10 \pm 0.36$ & $7.04 \pm 0.83$ & $6.81 \pm 0.60$ & $6.61 \pm 0.56$ & $6.35 \pm 1.27$ & $6.45 \pm 0.63$ & $7.02 \pm 0.43$ & $7.66 \pm 1.33$ & $7.48 \pm 1.05$ & $7.30 \pm 0.35$ \\
\hline \multirow[t]{2}{*}{$\mathrm{CHOL}$} & $\mathrm{mmol} / \mathrm{L}$ & Male & $1.20 \pm 0.26$ & $1.53 \pm 0.28$ & $1.63 \pm 0.21$ & $1.34 \pm 0.33$ & $1.44 \pm 0.35$ & $1.41 \pm 0.10$ & $1.58 \pm 0.26$ & $1.53 \pm 0.36$ & $1.73 \pm 0.13$ & $1.81 \pm 0.36$ & $1.46 \pm 0.10$ & $1.35 \pm 0.21$ \\
\hline & & Female & $1.77 \pm 0.38$ & $2.20 \pm 0.38$ & $1.97 \pm 0.47$ & $2.14 \pm 0.31$ & $2.33 \pm 0.42$ & $2.46 \pm 1.10$ & $2.23 \pm 0.39$ & $2.25 \pm 0.56$ & $2.20 \pm 0.46$ & $2.07 \pm 0.38$ & $2.08 \pm 0.44$ & $1.92 \pm 0.53$ \\
\hline \multirow[t]{2}{*}{ TG } & $\mathrm{mmol} / \mathrm{L}$ & Male & $0.50 \pm 0.06$ & $0.66 \pm 0.20$ & $0.74 \pm 0.73$ & $0.90 \pm 0.45$ & $0.84 \pm 0.36$ & $0.85 \pm 0.27$ & $0.84 \pm 0.36$ & $0.92 \pm 0.39$ & $0.65 \pm 0.16$ & $0.74 \pm 0.03^{* *}$ & $1.01 \pm 0.39^{*}$ & $0.74 \pm 0.41$ \\
\hline & & Female & $0.62 \pm 0.08$ & $1.18 \pm 0.62$ & $0.70 \pm 0.46$ & $0.70 \pm 0.13$ & $0.92 \pm 0.50$ & $0.85 \pm 0.28$ & $0.58 \pm 0.11$ & $0.51 \pm 0.12$ & $0.61 \pm 0.25$ & $0.84 \pm 0.32$ & $0.75 \pm 0.25$ & $0.59 \pm 0.20$ \\
\hline \multirow[t]{2}{*}{$\mathrm{TP}$} & $g / L$ & Male & $5.29 \pm 0.12$ & $5.36 \pm 0.29$ & $5.54 \pm 0.25$ & $5.34 \pm 0.25$ & $5.50 \pm 0.47$ & $5.45 \pm 0.18$ & $5.70 \pm 0.19^{* *}$ & $5.37 \pm 0.51$ & $5.50 \pm 0.19$ & $5.84 \pm 0.13^{* *}$ & $5.45 \pm 0.38$ & $5.67 \pm 0.22$ \\
\hline & & Female & $5.93 \pm 0.22$ & $6.34 \pm 0.27$ & $6.32 \pm 0.42$ & $6.31 \pm 0.48$ & $6.72 \pm 0.50$ & $6.81 \pm 0.33$ & $6.39 \pm 0.27^{*}$ & $6.37 \pm 0.43$ & $6.32 \pm 0.26$ & $6.48 \pm 0.26^{* *}$ & $6.49 \pm 0.50$ & $6.22 \pm 0.27$ \\
\hline \multirow[t]{2}{*}{ ALB } & $g / L$ & Male & $3.02 \pm 0.08$ & $2.83 \pm 0.34$ & $3.04 \pm 0.15$ & $2.99 \pm 0.21$ & $2.92 \pm 0.40$ & $3.12 \pm 0.13$ & $3.33 \pm 0.15^{* *}$ & $2.86 \pm 0.45$ & $3.18 \pm 0.06$ & $3.37 \pm 0.11^{* *}$ & $2.95 \pm 0.41$ & $3.09 \pm 0.13$ \\
\hline & & Female & $3.50 \pm 0.22$ & $3.63 \pm 0.30$ & $3.78 \pm 0.35$ & $3.69 \pm 0.28$ & $3.83 \pm 0.31$ & $4.00 \pm 0.15$ & $3.65 \pm 0.19$ & $3.64 \pm 0.30$ & $3.70 \pm 0.26$ & $3.87 \pm 0.22^{*}$ & $3.61 \pm 0.38$ & $3.71 \pm 0.17$ \\
\hline \multirow[t]{2}{*}{ T-BIL } & $\mu \mathrm{mol} / \mathrm{L}$ & Male & $3.4 \pm 0.4$ & $2.9 \pm 1.6$ & $3.0 \pm 2.5$ & $3.8 \pm 0.9$ & $3.0 \pm 0.7$ & $2.4 \pm 0.5$ & $3.1 \pm 0.9$ & $3.1 \pm 0.6$ & $2.0 \pm 0.4$ & $3.5 \pm 0.8$ & $2.9 \pm 1.2$ & $2.6 \pm 1.1$ \\
\hline & & Female & $3.9 \pm 0.5$ & $4.1 \pm 1.4$ & $3.0 \pm 0.8$ & $3.0 \pm 0.7$ & $3.6 \pm 0.8$ & $3.7 \pm 0.9$ & $3.7 \pm 1.0$ & $2.7 \pm 0.6^{*}$ & $2.7 \pm 0.5$ & $3.1 \pm 0.7$ & $3.9 \pm 0.8$ & $2.8 \pm 0.5$ \\
\hline \multirow[t]{2}{*}{ D-BIL } & $\mu \mathrm{mol} / \mathrm{L}$ & Male & $2.4 \pm 0.4$ & $1.4 \pm 0.5$ & $1.8 \pm 0.6$ & $2.2 \pm 0.6$ & $1.2 \pm 0.5$ & $1.7 \pm 0.2$ & $1.2 \pm 0.4$ & $1.0 \pm 0.4$ & $1.4 \pm 0.1$ & $1.3 \pm 0.6$ & $1.1 \pm 0.9$ & $1.6 \pm 0.5$ \\
\hline & & Female & $2.5 \pm 0.1$ & $2.0 \pm 1.0$ & $1.9 \pm 0.4$ & $1.5 \pm 0.3$ & $1.5 \pm 0.6$ & $2.5 \pm 0.4$ & $1.8 \pm 0.7$ & $0.9 \pm 0.4^{* *}$ & $1.9 \pm 0.3$ & $0.9 \pm 0.2$ & $1.4 \pm 0.9$ & $1.8 \pm 0.3$ \\
\hline \multirow[t]{2}{*}{ Cre } & $\mu \mathrm{mol} / \mathrm{L}$ & Male & $70.3 \pm 4.6$ & $63.6 \pm 9.5$ & $65.2 \pm 10.3$ & $74.7 \pm 9.0$ & $64.8 \pm 11.9$ & $56.1 \pm 4.8$ & $80.3 \pm 10.0$ & $62.9 \pm 10.3$ & $73.3 \pm 11.8$ & $80.5 \pm 4.6$ & $64.7 \pm 4.1$ & $76.2 \pm 2.7$ \\
\hline & & Female & $78.8 \pm 5.9$ & $68.2 \pm 8.1$ & $67.3 \pm 7.2$ & $79.4 \pm 5.2$ & $66.9 \pm 5.1$ & $65.5 \pm 3.4$ & $82.6 \pm 6.4$ & $67.1 \pm 5.7$ & $63.2 \pm 1.1$ & $78.8 \pm 8.9$ & $71.4 \pm 7.7$ & $66.7 \pm 5.3$ \\
\hline \multirow[t]{2}{*}{ CPK } & $U / L$ & Male & $3214 \pm 597$ & $335 \pm 141$ & $183 \pm 47$ & $767 \pm 931$ & $325 \pm 324$ & $150 \pm 46$ & $404 \pm 61$ & $218 \pm 119$ & $301 \pm 38$ & $509 \pm 220$ & $245 \pm 111$ & $362 \pm 130$ \\
\hline & & Female & $2032 \pm 1363$ & $244 \pm 61$ & $168 \pm 89$ & $801 \pm 616$ & $248 \pm 173$ & $231 \pm 123$ & $488 \pm 143$ & $277 \pm 133$ & $120 \pm 54$ & $264 \pm 51$ & $158 \pm 44$ & $111 \pm 34$ \\
\hline \multirow[t]{2}{*}{ Ure } & $\mathrm{mmol} / \mathrm{L}$ & Male & $4.85 \pm 0.26$ & $5.77 \pm 0.82$ & $5.17 \pm 1.00$ & $4.57 \pm 0.44$ & $5.53 \pm 1.22$ & $5.35 \pm 0.66$ & $4.23 \pm 0.65$ & $5.88 \pm 0.73$ & $5.93 \pm 0.39$ & $4.33 \pm 0.43$ & $5.66 \pm 1.08$ & $6.02 \pm 1.44$ \\
\hline & & Female & $5.44 \pm 0.49$ & $6.08 \pm 0.73$ & $6.32 \pm 0.89$ & $5.04 \pm 0.51$ & $6.04 \pm 0.92$ & $6.13 \pm 1.01$ & $5.42 \pm 0.59$ & $6.72 \pm 1.11$ & $6.45 \pm 0.67$ & $5.71 \pm 0.87$ & $6.74 \pm 1.31^{* *}$ & $6.24 \pm 1.12$ \\
\hline \multirow[t]{2}{*}{$\mathrm{Na}^{+}$} & $\mathrm{mmol} / \mathrm{L}$ & Male & $141.3 \pm 0.7$ & $141.8 \pm 1.4$ & $142.3 \pm 0.2$ & $141.0 \pm 0.4$ & $142.4 \pm 1.6$ & $142.3 \pm 1.2$ & $140.7 \pm 1.3$ & $141.2 \pm 0.7$ & $141.9 \pm 1.5$ & $139.8 \pm 0.7$ & $141.1 \pm 1.4$ & $141.1 \pm 0.8$ \\
\hline & & Female & $141.6 \pm 0.9$ & $140.0 \pm 1.9$ & $141.8 \pm 0.9$ & $140.9 \pm 1.0$ & $140.9 \pm 0.9$ & $143.0 \pm 0.6$ & $140.3 \pm 1.0$ & $141.0 \pm 1.7$ & $142.6 \pm 1.4$ & $139.4 \pm 1.0$ & $139.8 \pm 0.8$ & $142.5 \pm 0.7$ \\
\hline
\end{tabular}


Table 4 Serum biochemical values in rats orally treated with HZJW (Continued)

\begin{tabular}{|c|c|c|c|c|c|c|c|c|c|c|c|c|c|c|}
\hline \multirow[t]{2}{*}{$\mathrm{K}^{+}$} & $\mathrm{mmol} / \mathrm{L}$ & Male & $81 \pm 0.27$ & $3.95 \pm 0.27$ & $3.65 \pm 0.20$ & $5.02 \pm 0.43$ & $4.08 \pm 0.64$ & $3.50 \pm 0.28$ & $5.16 \pm 0.32$ & $4.15 \pm 0.70$ & $3.90 \pm 0.20$ & $5.13 \pm 0.12$ & $3.92 \pm 0.56$ & $4.09 \pm 0.27$ \\
\hline & & Female & $4.43 \pm 0.25$ & $3.86 \pm 0.33$ & $3.43 \pm 0.36$ & $4.80 \pm 0.34$ & $3.57 \pm 0.20$ & 0.08 & 4.60 & 7 & 21 & $4.99=$ & 02 & $=0.3$ \\
\hline \multirow[t]{2}{*}{$\mathrm{Cl}^{-}$} & $\mathrm{mmol} / \mathrm{L}$ & Male & $111.1 \pm 0.4$ & $108.7 \pm 2.9$ & $107.0 \pm 2.2$ & $110.3 \pm 0.9$ & $106.7 \pm 1.5$ & $109.5 \pm 1.6$ & $108.2 \pm 1.3$ & $108.0 \pm 2.9$ & $107.2 \pm 0.5$ & $106.4 \pm 1.6$ & $107.6 \pm 2.2$ & $106.5 \pm 1$ \\
\hline & & male & $10.3 \pm 3.3$ & $108.9 \pm 2.3$ & $109.8 \pm 1.6$ & $109.7 \pm 2.1$ & $110.7 \pm 1.4$ & $109.8 \pm 0.8$ & $109.8 \pm 1.6$ & $109.2 \pm 2.8$ & 1.2 & $107.3 \pm 1.2$ & 1.6 & $10.1-$ \\
\hline
\end{tabular}

Note: Aspartate aminotransferase activity (AST), alanine aminotransferase activity (ALT), alkaline phosphatase activity (ALP), albumin (ALB), total protein (TP), glucose (GLU), total cholesterol (CHOL), creatine

phosphokinase activity (CPK), total bilirubin (T-BIL), direct bilirubin (D-BIL), creatinine (CRE), triglycerides (TG), urea (Ure), sodium ions ( $\left.\mathrm{Na}^{+}\right)$, potassium ions $\left(\mathrm{K}^{+}\right)$, chlorideions $(\mathrm{Cl})$. Values are mean $+\mathrm{S} . \mathrm{D}$. $(n=5(4$ weeks), 10 (26 weeks) or recovery (30 weeks)/sex/dose). ${ }^{*} P<0.05 \&{ }^{* *} P<0.01$ compared with normal group. 
this ulcerogenesis model, the lesion index increased by aspirin was observed to be counteracted by pretreatment with HZJW of different dosages. The data obtained demonstrated that HZJW-treated groups displayed significant reduction in ulcerative lesion, as compared to their control counterparts. Based on the observations outlined, HZJW was in possession of good therapeutic action on the gastric ulcers. The protective effect of HZJW against the gastric damage might be due to possible encouragement of gastric mucosal defense and/or stimulation of endogeneous prostaglandins secretion. However, the precise mechanism underlying this specific action merited further exploration.

Eradication of $H$. pylori is an important objective in overcoming gastric diseases. H. pylori is considered the main etiological agent of human peptic ulcer, with a worldwide prevalence rate of about $40 \%$ in developed countries and over $80 \%$ in developing countries [37]. Indeed, half of all gastric ulcer cases are associated with infection by $H$. pylori $[38,39]$. Given the major role that $H$. pylori plays in the aetiology of peptic ulcers, its eradication is strongly recommended as key for the effective management of these pathologies [2]. In this investigation, the in vitro anti-H. pylori properties as well as in vivo $H$. pylori eradication activity of HZJW in infected mice was assessed. Rapid urease test (RUT), with its high sensitivity and specificity, is considered to be a reliable test for the initial diagnosis of $H$. pylori infection [40]. In the RUT assay, HZJW exhibited strong antagonistic activity against $H$. pylori. HZJW were able to significantly reduce the number of animals that presented a positive urease test, thus preventing the colonization of $H$. pylori in the stomach. The immunogold test and histopathological analysis further confirmed the in vivo anti-H. pylori activity of HZJW, in which HZJW could reduce the microorganism detection in histological sections, and thereby clearing $H$. pylori from the stomach of infected mice. All these results suggested the possible in vivo efficacy in eradication therapy for $H$. pylori, and this anti-H. pylori activity should contribute to the therapeutic effect of HZJW in treating gastrointestinal disorders.

A number of studies have shown that $H$. pylori eradication by antibiotic drugs (i.e., metronidazole, amoxicillin, and clarithromycin) is achieved by both systemic and topical action $[41,42]$. However, HZJW displayed stronger in vivo anti- $H$. pylori activity while weaker activity against $H$. pylori in vitro. This suggests that the anti-H. pylori capacity of HZJW might not result from the topical action but may take place by a systemic action that is attributed to the metabolic transformation of multi components in HZJW into active intermediates. It is known that clearance of $H$. pylori from the stomach of infected patients can be due to the direct topical activity of the ingested drugs at the gastric mucosal epithelium, and specially the secondary systemic therapeutic activity, which result from the back secretion and re-entry of the absorbed active principle from the basal to the apical side of the gastric epithelium. After absorption into the bloodstream, HZJW is postulated to be secreted, metabolized into active forms in the liver or during its trans-intestinal passage, from the basal side back to the apical surface of the gastric epithelium where $H$. pylori is located, thus improving the level of clinical treatment. However, further in-depth exploration was warranted.

The equilibrium between the therapeutic versus toxicological effects of a drug is a vital parameter in assessing its applicability in relation to pharmacological action [43]. As a part of this pharmacological study, HZJW was investigated for the acute and general toxicity in rodents. The data obtained in acute toxicity test by a procedure of fixed dose suggest that the oral $\mathrm{LD}_{50}$ of HZJW in mice was over $18.0 \mathrm{~g} / \mathrm{kg}$. Anatomical results presented the absence of abnormal organic damages in the dead mice. During the 6-month repeated-dose (1000, 2500, or $5000 \mathrm{mg} / \mathrm{kg} /$ day of HZJW) study, no drug-induced variations in clinical signs or in the ophthalmological, histopathological, hematological and blood biochemistry were observed in any of the test-article-treated groups compared to the recovery and control group. Throughout the experimental period, there were no significant changes noted in general behavior, skin effects, defecation and postural abnormalities in all animals. Although clinical indications like fur loss were observed in some rats, these symptoms generally occurred spontaneously in toxicity test due to systemic administration [44]. Additionally, these symptoms were infrequent and not dose-dependent. Therefore, this symptom was not considered to be a HZJW-induced abnormality. Some changes in other clinical observations, hematology, serum biochemistry, gross findings and organ weights were considered to be not dosedependent and treatment-related, incidental and within the range of normal biological variation [45]. In addition, no corresponding histopathological findings were observed in related organs. Therefore, these were not considered to be triggered by HZJW.

In the histopathological examination, some rats presented with anomalous pathologies but no significant differences were found in relation to treatment with HZJW. No consistent treatment-related histopathological abnormalities were found in rats of either sex. These observations are readily detected in clinically normal rats of the age and were considered spontaneous or incidental in nature. Therefore, these findings observed sporadically in both sexes of the treatment groups without a dose-response relationship were not considered to be changes triggered by the administration of HZJW, according to the comparison to the recovery and vehicle control group. Based on these results, the No Observed Adverse Effect Level (NOAEL) of 
HZJW was considered to be $5,000 \mathrm{mg} / \mathrm{kg} /$ day for both sexes, a dose equivalent to 50 times of normal human dose in clinical prescription. In view of the doses of the components consumed, HZJW was extrapolated to offer a wide margin of safety by oral route. However, since toxicity in animals and humans is genetically diverse and may respond differently, especially with respect to conditions of gastrointestinal disorders, additional toxicological assessment in other species, such as dogs, needs to be performed to evaluate the safety of HZJM, and careful observation should be also conducted in clinical practice.

\section{Conclusions}

Taken together the present results, the efficacy and safety of HZJW in healing peptic ulcer and combating H. pylori were demonstrated. The findings outlined corroborated their conventional indications, and contributed to their antiulcer pharmacological validation, lending more credence to clinical applications for the traditional treatment of stomach complaints symptomatic of peptic ulcer disease (PUD). Therefore, HZJW might have the potential for further development as a safe and effective alternative/complementary to conventional medication in treating gastrointestinal (GI) disorders.

\section{Additional file}

Additional file 1: Figure S1. HPLC Chromatogram of HZJW and its components. HZJW and its main compounds were subjected to HPLC. The chromatograms were obtained at $335 \mathrm{~nm}$. (A): HZJW without Coptidis Rhizoma Scutellarin; (B) HZJW without Scutellariae Barabtae Herba: (C) HZJW without Cynanchi Paniculati Radix et Rhizoma; (D) Standard mixture of three major compounds: (1) scutellarin (10.35min), (2) berberine (21.67 min), (3) paeonol (25.94 min);(E) HZJW.

\begin{abstract}
Abbreviations
TCM: Traditional Chinese Medicine; RUT: Rapid urease test; NSAID: Nonsteroidal anti-inflammatory drug; PUD: Peptic ulcer disease; MIC: Minimum inhibitory concentration; NOAEL: No Observed Adverse Effect Level; RBC: Red blood cell; HGB: Hemoglobin; HCT: Hematocrit; MCV: Mean corpuscular volume; MCH: Mean corpuscular hemoglobin; MCHC: Mean corpuscular hemoglobin concentration; RDW: Red cell distribution width; PLT: Platelets; MPV: Mean platelet volume; PDW: Platelet distribution width; WBC: White blood cell counts; RET: Reticulocytes; NE: Neutrophils; LYM: Lymphocytes; MON: Monocytes; EOS: Eosinophils; BAS: Basophils; PT: Prothrombin time; AST: Aspartate aminotransferase activity; ALT: Alanine aminotransferase activity; ALP: Alkaline phosphatase activity; ALB: Albumin; TP: Total protein; GLU: Glucose; CHOL: Total cholesterol; CPK: Creatine phosphokinase activity; T-BIL: Total bilirubin; DBIL: Direct bilirubin; CRE: Creatinine; TG: Triglycerides; Ure: Urea; $\mathrm{Na}^{+}$: Sodium ions; $\mathrm{K}^{+}$: Potassium ions; $\mathrm{Cl}^{-}$: Chlorideions.
\end{abstract}

\section{Competing interest}

The authors declare that there are no conflicts of interest.

\section{Authors' contributions}

All authors participated in the acquisition of data and revision of the manuscript. All authors conceived of the study, determined the design, interpreted the data and drafted the manuscript. All authors read and gave final approval for the version submitted for publication.

\section{Acknowledgements}

This work was supported by grants from Comprehensive Strategic Cooperation Project for Guangdong --- Chinese academy of sciences (No. 2011B090300046 \& 2011B090300045), Guangdong International Cooperation Projects in 2012, Guangdong Province (No. 2012B050300002), Science and Technological Program for Dongguan's Higher Education, Science and Research, and Health Care Institutions (No.2012105102009) and Science and Technology Innovation Platform for Cooperation of Industry, Academe and Research in Health Industry of Traditional Chinese Medicine (No. 2010BB091000001).

\section{Author details}

${ }^{1}$ School of Chinese Materia Medica, Guangzhou University of Chinese Medicine, Guangzhou 510006, P. R. China. ²Dongguan Mathematical Engineering Academy of Chinese Medicine, Guangzhou University of Chinese Medicine, Dongguan 523808, P. R. China. ${ }^{3}$ Shandong College of Traditional Chinese Medicine, Yantai 264100, P. R. China. ${ }^{4}$ Shenzhen ENT Institute, Shenzhen 518172, P. R. China.

Received: 18 December 2012 Accepted: 23 May 2013

Published: 30 May 2013

\section{References}

1. Rao CV, Saíram K, Goel RK: Experimental evaluation of Bacopamonniera on rat gastric ulceration and secretion. Indian J Physiol Pharmacol 2000, 44:335-441.

2. Soll $\mathrm{AH}$ : Consensus conference. Medical treatment of peptic ulcer disease. Practice guidelines. Practice Parameters Committee of the American College of Gastroenterology. J Am Med Assoc 1996, 275:622-629.

3. Marshall BJ, Goodwin CS, Warren JR, Murray R, Blincow ED, Blackbourn SJ, Phillips M, Waters TE, Sanderson CR: Prospective double-blind trial of duodenal ulcer relapse after eradication of Campylobacter pylori. Lancet 1988, 2:1437-1442.

4. Bighetti AE, Antonio MA, Kohn LK, Rehder VL, Foglio MA, Possenti A, Vilela L, Carvalho JE: Antiulcerogenic activity of a crude hydroalcoholic extract and coumarin isolated from Mikania laevigata Schultz Bip. Phytomedicine 2005, 12:72-77.

5. Ramakrishnan K, Salinas RC: Peptic ulcer disease. Am Fam Physician 2007, 76:1005-1012.

6. Hoffelner H, Rieder G, Haas R: Helicobacter pylori vaccine development: optimisation of strategies and importance of challenging strain and animal model. Int J Med Microbiol 2008, 298:151-159.

7. Kabir S: The current status of Helicobacter pylori vaccines: a review. Helicobacter 2007, 12:89-102.

8. Borrelli F, Izzo AA: The plant kingdom as a source of antiulcer remedies. Phytother Res 2000, 14:581-591.

9. Ananya C, Sirshendu C, Sandip KB: H. pylori-induced Gastric Ulcer: Pathophysiology and Herbal Remedy. Int J Biol Med Res 2012, 3:1461-1465.

10. Xing $\mathrm{HL}$ : Observation of clinical efficacy of Huang-zhu-jian-wei Fang (HZJW) on 89 cases of chronic superficial gastritis disease. Acta Chin Med Pharmacol 2011, 39:125-126.

11. Chen JP, Li YS, YU XF, Chen YL, Lin RF, He YH, Lin J, Su ZR: RP-HPLC simultaneous determi nation of three kinds of components in Huangzhu Jianwei tablets. Chin J Pharm Anal 2010, 30(11):2157-2159.

12. Li Y, Xu C, Zhang Q, Liu JY, Tan RX: In vitro anti- Helicobacter pylori action of 30 Chinese herbal medicines used to treat ulcer diseases. J Ethnopharmacol 2005, 98:329-333.

13. Lee HB, Lee HK, Kim JR, Ahn YJ: Anti-Helicobacter pylori Diarylheptanoid Identified in the Rhizome of Alpinia officinarum. J Korean Soc Appl Bi 2009, $52: 367-370$

14. Lu JS, Liu YQ, Li M, Li BS, Xu Y: Protective effects and its mechanisms of total alkaloids from Rhizoma Coptis Chinensis on Helicobacter pylori LPS induced gastric lesion in rats. China J Chin Mater Med 2007, 32:1333-1336.

15. Han YH, Lee KD, Lee DU: Anti-Helicobacter pylori Activity and StructureActivity Relationships of Berberine Derivatives. B Korean Chem Soc 2009, 30:3147-3149.

16. Mahady GB, Pendland SL, Stoia A, Chadwick LR: In vitro susceptibility of Helicobacter pylori to isoquinoline alkaloids from Sanguinaria canadensis and Hydrastis Canadensis. Phytother Res 2003, 17:217-221. 
17. China Pharmacopoeia Committee: Pharmacopoeia of the People's Republic of China (I), Pharmacopoeia of the People's Republic of China (IV). Beijing: Chemical Industry Press; 2010

18. Morimoto $Y$, Shimohara $K$, Oshima S, Sukamoro T: Effects of the new antiulcer agent KB-5492 on experimental gastric mucosal lesions and gastric mucosal defensive factors, as compared to those of terprenone and cimetidina. Jpn J Pharmacol 1991, 57:955-6053.

19. Nwafor PA, Okwuasaba FK, Binda LG: Antidiarrhoel and antiulcerogenic effects of methanolic extract of Asparagus pubescens root in rats. J Ethnopharmacol 2000, 72:421-427.

20. Boda M, Tan PV, Nyasse B: Rapid in vivo screening method for the evaluation of new anti-Helicobacter medicinal plant preparations. Afri J Tradit Complem 2006, 3:102-114.

21. The Organization of Economic Co-operation and Development (OECD): The OECD guideline for testing of chemicals: 420 Acute oral toxicity. Paris: OECD; 2001.

22. Rosidah X, Yam MF, Sadikun A, Ahmad M, Akowuah GA, Asmawi MZ: Toxicology evaluation of standardized methanol extract of Gynura procumbens. J Ethnopharmacol 2009, 123:244-249.

23. Seeff LB: Herbal hepatotoxicity. Clin Liver Dis 2007, 11:577-596.

24. Tang JL, Liu BY, Ma KW: Traditional Chinese medicine. Lancet 2008, 372:1938-1940.

25. Veiga-Junior VF, Pinto AC, Maciel MAM: Medicinal plants: safe cure? Quim Nova 2005, 28:519-528.

26. Saad B, Azaizeh H, Abu-Hijleh G, Said O: Safety of traditional Arab herbal medicine. Evid-based Compl Alt 2006, 3:433-439.

27. Debelle FD, Vanherweghem $\mathrm{J}$, Nortier $\mathrm{J}$ : Aristolochic acid nephro-pathy: a worldwide problem. Kidney Int 2008, 74:158-169.

28. Castro1 LS, Perazzo FF, Maistro EL: Genotoxicity testing of Ambelania occidentalis (Apocynaceae) leaf extract in vivo. Genet Mol Res 2009, 8(2):440-447.

29. Tan PV, Dimo T, Dongo E: Effects of methanol, cyclohexane and methylene chloride extracts of Bidens pilosa on various gastric ulcer models in rats. J Ethnopharmacol 2000, 73:415-421.

30. Klein-Junior LC, Santin JR, Niero R, DE Andrade SF, Cechinel-Filho V: The therapeutic lead potential of metabolites obtained from natural sources for the treatment of peptic ulcer. Phytochem Rev, 11:567-616.

31. Nasuti C, Gabbianelli R, Falcioni G, Cantalamessa F: Antioxidative and gastroprotective activities of anti-inflammatory formulations derived from chestnut honey in rats. Nutr Res 2006, 26:130-137.

32. Sumitra C, Yogesh B, Mital K: Protective effect of Polyalthia longifolia var. pendula leaves on ethanol and ethanol/ $\mathrm{HCl}$ induced ulcer in rats and its antimicrobial potency. Asian Pac J Trop Med 2011:673-679.

33. Moleiro FC, Andreo MA, De Santos RC, Moraes TDM, Rodrigues CM, Carli CB, Lopes FC, Pellizzon CH, Carlos IZ, Bauab TM, Vilegas W, Hiruma-Lima CA: Mouriri elliptica: Validation of gastroprotective, healing and anti-Helicobacter pylori effects. J Ethnopharmaco 2009, 123:359-368.

34. Wallace JL: Prostaglandins, NSAIDs, and gastric mucosal protection: why doesn't the stomach digest itself? Physiol Rev 2008, 88:1547-1565.

35. Malfertheiner P, Chan FKL, McColl KEL: Peptic ulcer disease. Lancet 2009, 374:1449-1461.

36. Wallace JL: Pathogenesis of NSAID-induced gastroduodenal mucosal injury. Best Pract Res Cl Ga 2001, 15:691-703.

37. Shi R, Xu S, Zhang H, Ding Y, Sun G, Huang X, Chen X, Li X, Yan Z, Zhang G Prevalence and risk factors for Helicobacter pylori infection in Chinese Populations. Helicobacter 2008, 13:157-165.

38. Ge Z, Taylor DE: Contribution of genome sequencing to understanding the biology of Helicobactor pylori. Annual Rev Microbiol 1999, 53:358-387.

39. Cave DR: Transmission and epidemiology of Helicobactor pylori. Am J Med 1996, 100:12-17

40. Vandana B, Vidya S: Rapid Urease Test to Diagnose Helicobacter Pylori Infection. JK Sci 2006, 8:86-88.

41. Adamek RJ, Wegener M, Opferkuch W, Ruhl GH: Successful Helicobacter pylori eradication: a systemic effect of antibiotics? Am J Gastroenterol 1993, 88:792-793.

42. Goddard AF, Jessa MJ, Barrett DA, Shaw PN, Idstrom JP, Cederberg C: Effect of omeprazole on the distribution of metronidazole, amoxicillin, and clarithromycin in human gastric juice. Gastroenterol 1996, 111:358-367.
43. Loomis TA, Hayes AW: Essentials of Toxicology. 4th edition. London: Academic Press Limited; 1996.

44. Greaves P: , Histopathology of Preclinical Toxicity Studies. 3rd edition Amsterdam: Elsevier Academic Press; 2007.

45. Derelanko MJ: The Toxicologist's Pocket Handbook. 2nd edition. UK: Informa Healthcare; 2008.

doi:10.1186/1472-6882-13-119

Cite this article as: Xie et al.: Gastroprotective and anti-Helicobacter pylori potential of herbal formula HZJW: safety and efficacy assessment. BMC Complementary and Alternative Medicine 2013 13:119.

\section{Submit your next manuscript to BioMed Central and take full advantage of:}

- Convenient online submission

- Thorough peer review

- No space constraints or color figure charges

- Immediate publication on acceptance

- Inclusion in PubMed, CAS, Scopus and Google Scholar

- Research which is freely available for redistribution

Submit your manuscript at www.biomedcentral.com/submit
C Biomed Central 